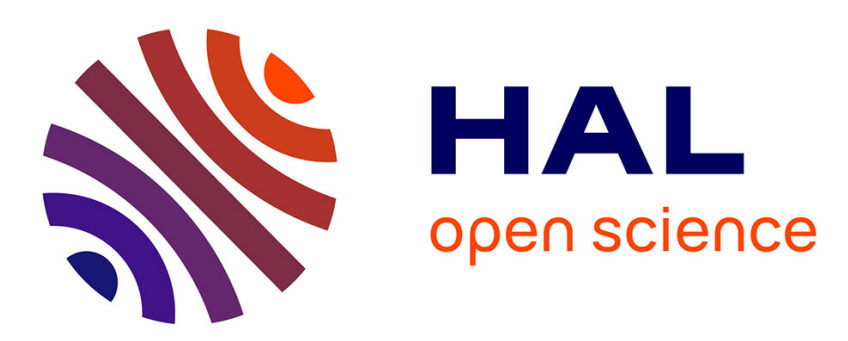

\title{
Large eddy simulation of explosion deflagrating flames using a dynamic wrinkling formulation
}

Pedro S Volpiani, Schmitt Thomas, Olivier Vermorel, Pierre Quillatre, Denis Veynante

\section{- To cite this version:}

Pedro S Volpiani, Schmitt Thomas, Olivier Vermorel, Pierre Quillatre, Denis Veynante. Large eddy simulation of explosion deflagrating flames using a dynamic wrinkling formulation. Combustion and Flame, 2017, 186, pp.17 - 31. 10.1016/j.combustflame.2017.07.022 . hal-01643051

\section{HAL Id: hal-01643051 https://hal.science/hal-01643051}

Submitted on 22 Nov 2021

HAL is a multi-disciplinary open access archive for the deposit and dissemination of scientific research documents, whether they are published or not. The documents may come from teaching and research institutions in France or abroad, or from public or private research centers.
L'archive ouverte pluridisciplinaire HAL, est destinée au dépôt et à la diffusion de documents scientifiques de niveau recherche, publiés ou non, émanant des établissements d'enseignement et de recherche français ou étrangers, des laboratoires publics ou privés. 


\title{
Large eddy simulation of explosion deflagrating flames using a dynamic wrinkling formulation
}

\author{
P. S. Volpiani $\dagger$, T. Schmitt $\dagger$, O. Vermorel $\ddagger$, P. Quillatre $\ddagger$, D. Veynante $\dagger$ \\ † Laboratoire EM2C, CNRS, Ecole CentraleSupélec, Université Paris-Saclay, \\ Bâtiment Eiffel, 3 rue Joliot-Curie, 91190 Gif-sur-Yvette, France \\ $\ddagger$ CERFACS, 42 Avenue Gaspard Coriolis, 31057 Toulouse, France
}

\begin{abstract}
Reliable predictions of flames propagating in a semi-confined environment are vital for safety reasons, once they are representative of accidental explosion configurations. Large eddy simulations of deflagrating flames are carried out using a dynamic flame wrinkling factor model. This model, validated from a posteriori analysis, is able to capture both laminar and turbulent flame regimes. At early stages of the flame development, a laminar flame propagates in a flow essentially at rest and the model parameter is close to zero, corresponding to a unity-wrinkling factor. Transition to turbulence occurs when the flame interacts with the flow motions generated by thermal expansion and obstacles. The model parameter and wrinkling factor take higher values at these stages. Three configurations investigated experimentally by Masri et al. (2012) corresponding to different scenarios of flame acceleration are simulated. The first case (OOBS) is characterized by a long laminar phase. In the second one (BBBS) the flame is the most turbulent and the highest overpressure is observed in the vessel. For the last case (BOOS), the flame front is relaminarized after crossing the first row of obstacles. In
\end{abstract}


all configurations, large eddy simulations (LES) predict the flow dynamics and maximum overpressure with good accuracy.

Keywords: Explosion, Dynamic modeling, Turbulent combustion, Large eddy simulation, Thickened flame model

\section{Introduction}

Accidental explosions of flammable gases are a current issue in process industries. Selecting the optimal conditions and parameters in the design and operation of chemical, petrochemical, mining, nuclear and others industrial plants is not only a matter of safety but also economical and environmental issues. In gas explosions of a premixed gas cloud, the pressure increase is governed by a complex unsteady interaction between flame propagation, turbulence and geometry. This overpressure is often considered as the key parameter, since it controls the severity of the explosion and corresponding damages. This complex phenomenon is very challenging for computational fluid dynamics (CFD) problems since it involves a large spectrum of spatial and time scales and encompasses a large range of flow and combustion regimes.

The typical research configuration used to study explosions in buildings consist in vessels with obstacles filled with a premixed flammable mixture. After the ignition, a laminar flame propagates in a flow essentially at rest. Transition to turbulence takes place when the flame starts to interact with obstacles and their wakes. This interaction strongly influences the shape of the flame front, the burning rate and, as a consequence, the overpressure. This flame induced flow field increases turbulence and combustion intensity, 
leading to flames, which can propagate at 100 to $200 \mathrm{~m} / \mathrm{s}$. In the worst scenario, the initial flame can transition to detonation and cause the destruction of the whole building.

A large number of experiments have been carried out in order to understand flame/turbulence interactions in vented explosion chambers with solid obstacles [1-8]. Effects of geometry [1-4] and fuel type [2-4] have been analyzed in order to point out mechanisms involved in the overpressure generation. Flame acceleration and deflagration to detonation transition are also subjects of several reviews [6-8]. This work focuses on the configurations studied experimentally by Masri and co-workers [2, 4]. Different geometries, fuel types and scales were reported. The experiments have access to the pressure evolution inside the chamber, the flame speed and flame front position along the middle section of the chamber. Additionally, they have well prescribed initial and boundary conditions and for this reason are very appropriate for model validation.

On the numerical side, thanks to the growing computational power and the availability of parallel computing algorithms, large eddy simulation (LES) is becoming a routinely used tool to predict and reproduce turbulent reactive flows [9-12]. In LES, the large turbulent structures of the flow are resolved and the effect of small structures that exhibit a more universal behavior are modeled. Unfortunately, chemical reactions in combustion processes occur at characteristic scales that are smaller than the mesh resolution and a good combustion model is vital to capture the physics of the flow. Several numerical studies dealing with deflagrations in semi-confined chambers are reported in the literature [13-25]. 
The majority of LES of premixed flame deflagration in the presence of obstacles relies on the Flame Surface Density (FSD) approach. In this case, the species transport equations are simplified in the form of a transport equation for the reaction progress variable, which is zero within fresh reactants and unity within burned products [13-24]. The only exception is Quillatre et al. [25] that use the Thickening Flame model for LES (TFLES) together with reduced kinetic schemes for $\mathrm{CH}_{4}-\mathrm{C}_{3} \mathrm{H}_{8}$ and $\mathrm{H}_{2}$ /air combustion. While the former method has the advantage of being computationally cheaper, the latter one takes into account molecular and thermal transports, which turn out to have a significant impact on the results [25].

Di Sarli et al. [16] highlighted the importance of the sub-grid closure in the reproduction of the experiment, studied by Patel et al. [26], in terms of flame acceleration and deceleration around each obstacle, flame shape and speed as well as pressure peak. Actually, most of the models employed in their study needed parameter adjustments to obtain more realistic trends. Di Sarli et al. [21-23] also carefully validated LES FSD algebraic models of explosion deflagrating flames against precise time- and space-resolved experimental data including images of the propagating flame front and velocity vector maps. They note that, when the grid resolution is of the order of the laminar flame thickness, numerical results correctly match experiments even without sub-grid scale combustion model [21] but the generation of turbulence induced by the thermal expansion is mainly limited to a large scale vortex in their configuration.

As a matter of fact, LES models based on algebraic expressions for the turbulent flame speed [27, 28], the flame surface density [29] or the flame 
surface wrinkling factor $[30,31]$ are derived assuming local equilibrium between flame surface and turbulence motions. Model expressions depend on local turbulence characteristics such as turbulence intensity and length scales evaluated during simulations. Reaction rates then vary with space and time but may be erroneous when equilibrium is not reached, requiring the adjustment of model parameters for any small change in the operating conditions or in the geometry [32]. Models based on a balance equation for the flame surface density [33-35] or the wrinkling factor measuring the ratio of total and resolved flame surfaces [36], release this equilibrium assumption by construction but require additional closures. In practice, some model coefficients may still have to be adjusted when operating conditions change. For example, Mouriaux et al. [37] adjusted one parameter in the flame surface density transport equation when varying internal combustion engine speed.

Dynamic models, which take advantage of the known resolved large scales to automatically adjust model parameters, are an attractive alternative for these situations. The model is written at LES and test-filtered scales and the parameter is the solution of a Germano-like equation [38]. Validated in simple flow configurations (flame embedded in a homogeneous isotropic turbulence) [39-41], dynamic models have proven to be very effective in reproducing steady stable [42-46] and unstable [47] flames. They were successfully employed to simulate transient phenomena as well. Mouriaux et al. [37] obtained very good results, when dealing with internal combustion engines at distinct speeds. Gubba et al. [19], Ibrahim et al. [20] obtained accurate predictions using the dynamic FSD similarity formulation developed by Knikker et al. [48] for various explosion configurations studied experimentally 
by Masri et al. [4].

This work aims to validate the local dynamic wrinkling factor approach coupled with the Thickened Flame (TFLES) combustion model in the explosion test-cases studied experimentally by Masri et al. [4]. This combination has already been employed in other studies [39, 43, 46, 47]. The present manuscript is organized as follows: basic concepts of TFLES and the dynamic approach are discussed in Section 2. In Section 3, the experimental test cases are presented. Then, the chemical scheme employed in our computations is presented (Section 4). In Section 5, a sensitivity analysis, regarding mesh sizes, transport models, boundary conditions and other features of numerical simulations, is carried out on 2D-DNS and 2D-LES configurations and serves as basis for the three-dimensional study. The numerical set-up for the three-dimensional configurations is introduced in Section 6. Finally, a posteriori results for different geometries, corresponding to three scenarios of flame acceleration, are discussed (Section 7). Conclusions are drawn.

\section{Modeling}

\subsection{The thickened flame model (TFLES)}

One of the challenges in large eddy simulations of premixed combusting flows is the fact that the flame front cannot be resolved on the computational mesh. A common procedure to overcome this problem is to artificially thicken the flame by multiplying diffusion and dividing reaction rates by a thickening factor $\mathcal{F}[49]$. The modified flame front of thickness $\mathcal{F} \delta_{L}^{0}$ propagates at the same laminar flame speed $S_{L}$ as the original flame of thickness $\delta_{L}^{0}$. However, the Damköhler number is modified and the flame becomes less sensitive to 
turbulence [30]. A wrinkling factor $\Xi_{\Delta}$ is then introduced to counterbalance the reduction of flame surface induced by the thickening operation [30, 31]. The balance equations for filtered species mass fractions $\widetilde{Y}_{k}$ are written as:

$$
\frac{\partial \bar{\rho} \widetilde{Y}_{k}}{\partial t}+\nabla \cdot\left(\bar{\rho} \widetilde{\mathbf{u}} \widetilde{Y}_{k}\right)=-\nabla \cdot\left(\Xi_{\Delta} \mathcal{F} \overline{\rho \mathbf{V}_{k}} \widetilde{Y}_{k}\right)+\frac{\Xi_{\Delta}}{\mathcal{F}} \dot{\omega}_{k}(\widetilde{Q})
$$

where $\rho$ is the density, $\mathbf{u}$ the velocity vector, $\mathbf{V}_{\mathbf{k}}$ the diffusion velocity of species $k$, expressed here using the Hirschfelder and Curtiss approximation [11] and $\dot{\omega}_{k}$ the reaction rate of species $k$. Any quantity $\bar{Q}$ corresponds to the filtered Q-field, while $\widetilde{Q}=\overline{\rho Q} / \bar{\rho}$ denotes mass-weighted filtering. Equation (1) propagates a flame front of thickness $\mathcal{F} \delta_{L}^{0}$ at the sub-grid scale turbulent velocity $S_{T}=\Xi_{\Delta} S_{L}$. The balance equation for the filtered total energy is modified in the same manner.

\subsection{Dynamic wrinkling model}

The wrinkling factor $\Xi_{\Delta}$ models the ability of sub-grid scale vortices to wrinkle the flame front. This term is usually modeled in the literature by complex algebraic expressions. Generally, these expressions involve other unresolved terms (for instance, the sub-grid scale turbulent velocity, $u_{\Delta}^{\prime}$ ), which also need modeling. The sub-grid scale wrinkling factor $\Xi_{\Delta}$ is modeled as:

$$
\Xi_{\Delta}=\left(\frac{\Delta}{\delta_{c}}\right)^{\beta}
$$

where $\beta$ is the model parameter. In the present work, the inner cut-off $\delta_{c}$ (i. e. the smallest scale for the interaction of turbulent eddies with the premixed flame front) is identified to the laminar flame thickness, an assumption validated using two-dimensional simulations (see Subsection 5.6) 
and in agreement with other studies $[39,50]$. When $\beta$ is independent on the scale $\Delta$ and verifies $0 \leq \beta \leq 1$, Eq. (2) recover the fractal model [51-53], where $D=\beta+2$ is the fractal dimension of the flame surface. Herein, the present formulation is more general: $\beta$ may take here any value and vary both with time and location in the flow to describe complex surfaces [31].

The model parameter $\beta$ is dynamically determined equating flame surfaces computed at the test-filtered level and using test-filtered variables $[40,45,50]$ :

$$
\left\langle\Xi_{\Delta}|\nabla \widetilde{c}|\right\rangle=\left\langle\Xi_{\gamma \Delta}|\nabla \widehat{\widetilde{c}}|\right\rangle
$$

$c$ stands for the progress variable, increasing from 0 in fresh to 1 in burnt gases and is computed here from temperature. ${ }^{1}$ The hat symbol denotes a test-filtering operator. The effective filter size is $\gamma \Delta$, with $\gamma=\left[1+(\widehat{\Delta} / \Delta)^{2}\right]^{1 / 2}$ when combining two Gaussian filters of width $\Delta$ and $\widehat{\Delta}$. Symbol $\langle\cdot\rangle$ denotes an averaging operator over a given volume. In the current work, this operation is replaced by a Gaussian filter $\Delta_{\text {avg }}$, easier to implement as unsteady diffusion operators [54] when using unstructured meshes and a parallel solver [50].

Combining Eqs (2) and (3) and assuming that $\beta$ is identical at scales $\Delta$

\footnotetext{
${ }^{1}$ In vented explosions, reference fresh, $T_{u}$, and fully burnt, $T_{b}$, gas temperatures entering the estimation of the progress variable from the temperature $T$ as $c=\left(T-T_{u}\right) /\left(T_{b}-T_{u}\right)$ are expected to vary with pressure. These variations are not taken into account here as the dynamic model involves the ratio of progress variable gradients only (see Eq. 4). $T_{u}$ and $T_{b}$ then vanish in the computation of $\beta$ that could be directly estimated from temperature gradients.
} 
and $\gamma \Delta$ and constant over the averaging domain $\langle$.$\rangle give:$

$$
\beta=\frac{\log (\langle|\widehat{\nabla \widetilde{c}}|\rangle /\langle|\nabla \widehat{\widetilde{c}}|\rangle)}{\log (\gamma)}
$$

A thickened flame is not strictly equivalent to a filtered flame. Here, an equivalent filter size, $\Delta$, is estimated as a function of the thickening factor and the laminar flame thickness as $\Delta \approx \mathcal{F} \delta_{L}^{0}$ [31]. Test and averaging filter sizes are constant and set to $2 \Delta$ and $2.7 \Delta$ respectively. This choice results from extensive test validations [46] showing that $\beta$ is not sensitive to $\widehat{\Delta}$, as long as not too large, while increasing $\Delta_{a v g}$ reduces its variance [see also 47].

As the code time step is based on the acoustic CFL number and the model parameter is expected to evolve with convective times, the dynamic procedure is not applied every time step. This technique saves computational cost linked to the filtering operation. A simple analysis that compute the flame convective characteristic time based on the ratio of test filter size and maximum velocity shows that updating the model parameter every 100 iterations is sufficient. $^{2}$ Note that this artifice used to save computational time is no longer valid when using the dynamic flame surface density (DFSD) formulation [19, 20] developed by Knikker et al. [48]. As a matter of fact, the DFSD model is based on a similarity assumption and needs a filtering operation every time step, not only to compute $\beta$, but also the flame surface $\Sigma:$

\footnotetext{
${ }^{2}$ Assuming a characteristic length scale equal to the test filter size $\widehat{\Delta}=3.4 \mathrm{~mm}$, a maximum velocity about $150 \mathrm{~m} / \mathrm{s}$ and a time step of $0.5 \times 10^{-7} \mathrm{~s}$, a value of 400 iterations is found.
} 


$$
\Sigma=\Xi_{\Delta}|\nabla \bar{c}|=|\nabla \bar{c}|+\frac{1}{1-\gamma^{-\beta}}\left[\left(\frac{\Delta}{\delta_{c}}\right)^{\beta}-1\right][|\widehat{\nabla \bar{c}}|-|\nabla \widehat{\bar{c}}|]
$$

In Eq. (5), the term $|\widehat{\nabla \bar{c}}|-|\nabla \widehat{\bar{c}}|$ needs to be test-filtered every iteration. For this reason, using Knikker et al. [48] model in a compressible CFD solver, as done by Gubba et al. [19], Ibrahim et al. [20] for the same configuration, may be extremely expensive ${ }^{3}$ and the present methodology is preferred.

\section{Experimental setup}

Different geometries of a small scale combustion chamber studied experimentally by Masri and co-workers [4] are investigated in this work. The experiment is composed of a square cross section combustion chamber $(5 \mathrm{~cm}$ $\times 5 \mathrm{~cm} \times 25 \mathrm{~cm})$ with solid obstacles. The geometry is detailed in Fig. 1 with dimensions in millimeters. The left side of the chamber is closed, while the right side is opened out to the atmosphere. Removable baffle plates can be placed at various distances from the ignition source. Each one consists of five strips, $4 \mathrm{~mm}$ wide, equally separated by six gaps, $5 \mathrm{~mm}$ wide and they are placed 20, 50 and $80 \mathrm{~mm}$ from the closed end. Downstream of the baffle plates, an obstacle of cross section $12 \times 12 \mathrm{~mm}^{2}$ is placed at $94 \mathrm{~mm}$ from the ignition point.

The fuel-air mixture is initially at rest and is ignited by focusing a laser at the closed end of the chamber. Even though, different fuels are available, a focus is made on the liquefied petroleum gas LPG $\left(95 \% \mathrm{C}_{3} \mathrm{H}_{8}, 4 \% \mathrm{C}_{4} \mathrm{H}_{10}, 1 \%\right.$ $C_{5+}$ hydrocarbons by volume) at equivalence ratio $\phi=1$. The experimental

\footnotetext{
${ }^{3} \mathrm{~A}$ simple computation indicates that the $\mathrm{CPU}$ time is multiplied by a factor $\approx 20$.
} 
database includes pressure-time measurements and flame front visualizations based on high-speed laser induced fluorescence OH (LIF-OH) imaging.

Figure 2 illustrates the three cases studied: BBBS, OOBS and BOOS. The nomenclature from [4] is kept, where B indicates the presence of a baffle plate, O indicates its absence and $\mathrm{S}$ stands for the small central obstacle located after the grids. The three configurations correspond to distinct scenarios

of flame acceleration. In configuration BBBS, the transition takes place in the early stages of the flame development, resulting in a strong overpressure. Case OOBS is characterized by a long laminar phase, since obstacles are placed far away of the ignition point. In case BOOS, after passing the first baffle, the flame is relaminarized before reaching the central obstacle. This set of configurations seems appropriate to validate the dynamic combustion model, once the flame characteristics strongly differ from case to case.

\section{Chemical scheme}

The liquefied petroleum gas will be identified to pure propane in the following. Detailed chemistry mechanisms for propane/air combustion such as GRI-MECH 3.0 [55] involve more than 50 species and 350 reactions and, as a result, their implementation in turbulent flame simulations turns out to be prohibitive. A solution is to use reduced chemical schemes that reproduce the same laminar flame speed and burnt gas temperature as the detailed one. The two-step reduced chemical mechanism developed by Quillatre et al. [25] is retained here: 


$$
\begin{aligned}
\mathrm{C}_{3} \mathrm{H}_{8}+3.5 \mathrm{O}_{2} & \longrightarrow 3 \mathrm{CO}+4 \mathrm{H}_{2} \mathrm{O} \\
\mathrm{CO}+0.5 \mathrm{O}_{2} & \longleftrightarrow \mathrm{CO} 2
\end{aligned}
$$

The corresponding reaction rate expressions are given by:

$$
\begin{aligned}
& q_{1}=A_{1} \exp \left(\frac{-E_{a 1}}{R T}\right)\left(\frac{\rho Y_{C_{3} H_{8}}}{W_{C_{3} H_{8}}}\right)^{n_{C_{3} H_{8}}^{1}}\left(\frac{\rho Y_{O_{2}}}{W_{O_{2}}}\right)^{n_{O 2}^{1}} \\
& q_{2}=A_{2} \exp \left(\frac{-E_{a 2}}{R T}\right)\left[\left(\frac{\rho Y_{C O}}{W_{C O}}\right)^{n_{C O}^{2}}\left(\frac{\rho Y_{O_{2}}}{W_{O_{2}}}\right)^{n_{O 2}^{2}}-\frac{1}{K_{e}(T)}\left(\frac{\rho Y_{C O_{2}}}{W_{C O_{2}}}\right)^{n_{C O_{2}}^{2}}\right]
\end{aligned}
$$

where the pre-exponential factor, the activation energy and the model exponents are summarized on Table 1 for each equation. $K_{e}(T)$ is the equilibrium constant for the second reaction. The corresponding laminar flame properties are listed in Table 2.

The Lewis number $L e_{k}$ of species $k$ measures the competition between thermal and mass diffusion effects: $L e_{k}=D_{T} / D_{k}$, where $D_{T}$ and $D_{k}$ denote respectively the thermal and mass diffusivities. Assuming unity Lewis numbers is common in reactive CFD codes, but this simplification not only imposes the same thermal and mass diffusivities for all species but also strongly modifies the flame response to stretch. Actually, in the limit of small stretches, the consumption speed $S_{C}$ (defined as the integral of the fuel burning rate across the flame front) is given by $[56,57]$ :

$$
\frac{S_{C}}{S_{L}}=1-\mathcal{L}_{a}^{c} \frac{\kappa}{S_{L}}
$$




\begin{tabular}{llll}
\hline \hline $\mathrm{n} \quad$ Coefficients & $\mathrm{A}[$ cgs units $]$ & $E_{a}[\mathrm{cal} / \mathrm{mol}]$ \\
$1 \quad n_{\mathrm{C}_{3} \mathrm{H}_{8}}^{1}=0.55 n_{\mathrm{O}_{2}}^{1}=0.9$ & $1.33 \times 10^{12}$ & $4.15 \times 10^{4}$ \\
2 & $n_{\mathrm{CO}}^{2}=1.0 n_{\mathrm{O}_{2}}^{2}=0.5$ & $4.5 \times 10^{10}$ & $2.0 \times 10^{4}$ \\
& $n_{\mathrm{CO} O_{2}}^{2}=1.0$ & & \\
&
\end{tabular}

Table 1: Two-step reduced chemical mechanism for $\mathrm{C}_{3} H_{8}-A i r$. Coefficients for the Arrhenius formulation [25].

\begin{tabular}{lll}
\hline \hline & & \\
$S_{L}[\mathrm{~m} / \mathrm{s}]$ & $\delta_{L}^{0}[\mathrm{~mm}]$ & $T_{b}[\mathrm{~K}]$ \\
0.383 & 0.341 & 2300 \\
& & \\
\hline
\end{tabular}

Table 2: Laminar flame properties for the given mixture at equivalence ratio $\phi=1.0, P_{a t m}=101325 \mathrm{~Pa}$ and $T_{a t m}=300 \mathrm{~K} . S_{L}, \delta_{L}^{0}$ and $T_{b}$ stand for the laminar flame speed, the laminar flame thickness based on the temperature gradient and the burnt gas temperature, respectively. 
where $\kappa=(1 / S) d S / d t$ is the flame stretch, $\mathrm{S}$ being the flame surface area. $\mathcal{L}_{a}^{c}$ is the Markstein length and is linked to the Lewis number through the following expression [58]:

$$
\mathcal{L}_{a}^{c}=\frac{1}{2} \zeta \delta_{L}^{0}\left(L e_{F}-1\right) \frac{T_{f}}{T_{b}-T_{f}} \int_{0}^{\left(T_{b}-T_{f}\right) / T_{f}} \frac{\ln (1+x)}{x} d x
$$

where $T_{b}$ and $T_{f}$ are the burnt and fresh gas temperatures, respectively, and $\delta_{L}^{0}$ is the unstretched flame thickness. The parameter $\zeta=\left(T_{b}-T_{f}\right) T_{a} / T_{b}^{2}$ measures the activation energy, $T_{a}$ being the activation temperature $\left(T_{a}=\right.$ $E_{a} / R, R$ being the perfect gas constant).

Equations (10) and (11) show that the Lewis number has a direct influence on the consumption speed when the flame is stretched. The consumption speed of a fuel with $L e>1\left(C_{3} H_{8}\right.$ for instance) will be significantly reduced for high curvatures, as typically encountered in the early times following ignition [25].

The assumption of a unity Lewis number is too restrictive for the present configuration as shown in [59]. Moreover, the thickening operation introduced by the TFLES model affects the flame response to stretch through the flame thickness entering Eq. (11), reducing the consumption speed and the burnt gas temperature. This issue is discussed in details by Quillatre [60]. To overcome this problem, the author proposed to keep the quantity $\delta_{L}^{0}\left(L e_{k}-1\right)$ constant. Therefore, when using the TFLES model, the Lewis number is modified $\left(L e_{K}^{*}\right)$ to preserve the flame response to stretch:

$$
\mathcal{F} \delta_{L}^{0}\left(L e_{k}^{*}-1\right)=\delta_{L}^{0}\left(L e_{k}-1\right)
$$

giving: 


$$
L e_{k}^{*}=1+\frac{L e_{k}-1}{\mathcal{F}}
$$

In our simulations, a constant Prandtl number is specified $(\operatorname{Pr}=0.68$ herein), meaning that Schmidt numbers become:

$$
S c_{k}^{*}=\operatorname{Pr}+\frac{S c_{k}-P r}{\mathcal{F}}
$$

The proposed procedure is verified and validated by simulating a cylindrical 2D laminar flame using AVBP, which is representative of the first moments after ignition. Figure 3 shows the normalized consumption speed as a function of the flame radius for different thickening factors with and without correction. The original flame $(\mathcal{F}=1)$ speed tends towards the unstrained flame speed value when the flame radius increases and the stretch rate acting on the flame surface decreases. The unstrained flame speed value is correctly predicted, even for very low flame radii, when setting Lewis numbers to unity (not shown in Fig. 3 for clarity). When the flame is thickened, the effect of stretching on the flame is amplified and its consumption speed is drastically decreased while discrepancies are remarkably reduced when Lewis (Eq. 13) and Schmidt (Eq. 14) numbers are corrected. However, we note in Fig. 3 that the flame speed does not tend, or very slowly, towards the unstrained laminar flame speed for $\mathcal{F}=4$ and $\mathcal{F}=8$ with adapted Lewis numbers (underestimation of about $5 \%$ for a radius of $0.04 \mathrm{~m}$ ). This point is today not fully understood and remains to be investigated in details. Anyway, the adaptation of Lewis and Schmidt numbers with $\mathcal{F}$ retained here strongly improves the prediction of the thickened flame model.

Table 3 gives real $(\mathcal{F}=1)$ and modified $(\mathcal{F}=5$, Eq. 13) Lewis numbers. 


\begin{tabular}{lllllll}
\hline \hline & & & & & & \\
Lewis number/species & $\mathrm{CH}_{4}$ & $\mathrm{H}_{2} \mathrm{O}$ & $\mathrm{CO}_{2}$ & $\mathrm{CO}$ & $\mathrm{O}_{2}$ & $N_{2}$ \\
Real (EGLIB [61]) & 1.825 & 0.8 & 1.39 & 1.103 & 1.087 & 1.068 \\
Adapted $(\mathcal{F}=5)$ & 1.165 & 0.96 & 1.08 & 1.020 & 1.017 & 1.014 \\
& & & & & & \\
\hline \hline
\end{tabular}

Table 3: Table of real $(\mathcal{F}=1)$ and modified $(\mathcal{F}=5$, Eq. 13) Lewis numbers.

\begin{tabular}{lllllll}
\hline \hline & & & & & & \\
Schmidt number/species & $\mathrm{CH}_{4}$ & $\mathrm{H}_{2} \mathrm{O}$ & $\mathrm{CO}_{2}$ & $\mathrm{CO}$ & $\mathrm{O}_{2}$ & $\mathrm{~N}_{2}$ \\
Real (EGLIB [61]) & 1.241 & 0.544 & 0.945 & 0.750 & 0.739 & 0.726 \\
Adapted $(\mathcal{F}=5)$ & 0.792 & 0.653 & 0.733 & 0.694 & 0.692 & 0.689 \\
& & & & & & \\
\hline \hline
\end{tabular}

Table 4: Table of real $(\mathcal{F}=1)$ and modified $(\mathcal{F}=5$, Eq. 14) Schmidt numbers.

Corresponding Schmidt numbers, computed from Eq. (14), can be found in Table 4 . 


\section{Preliminary results on a two-dimensional configuration}

In this work, the massively parallel AVBP solver [62] is employed to solve the unsteady compressible and reactive multi-species Navier-Stokes equations on unstructured grids. The main advantage of $2 \mathrm{D}$ simulations is that they do not demand huge computational resources and a sensitivity analysis is easily performed. The authors are aware of the limitations introduced by two-dimensional simulations, which does not allow direct comparison with experiments. The only objective of this simple test case is to clarify important points and numerical constraints. The influence of the following parameters are investigated:

- boundary conditions

- mesh resolution

- numerical scheme

- inner cut-off length scale $\delta_{c}$

- transport modeling

\subsection{Numerical set-up for the $2 D$ configuration}

The computational domain is $250 \mathrm{~mm}$ long and $50 \mathrm{~mm}$ wide. Only the most turbulent geometry, BBBS (three ranges of baffle plates and a square section obstacle), is discussed.

Table 5 indicates all bi-dimensional test-cases studied with their respective numerical conditions, while Table 6 summarizes the five grids employed to run the simulations. First of all, the 2D-DNS are analyzed. The influence 


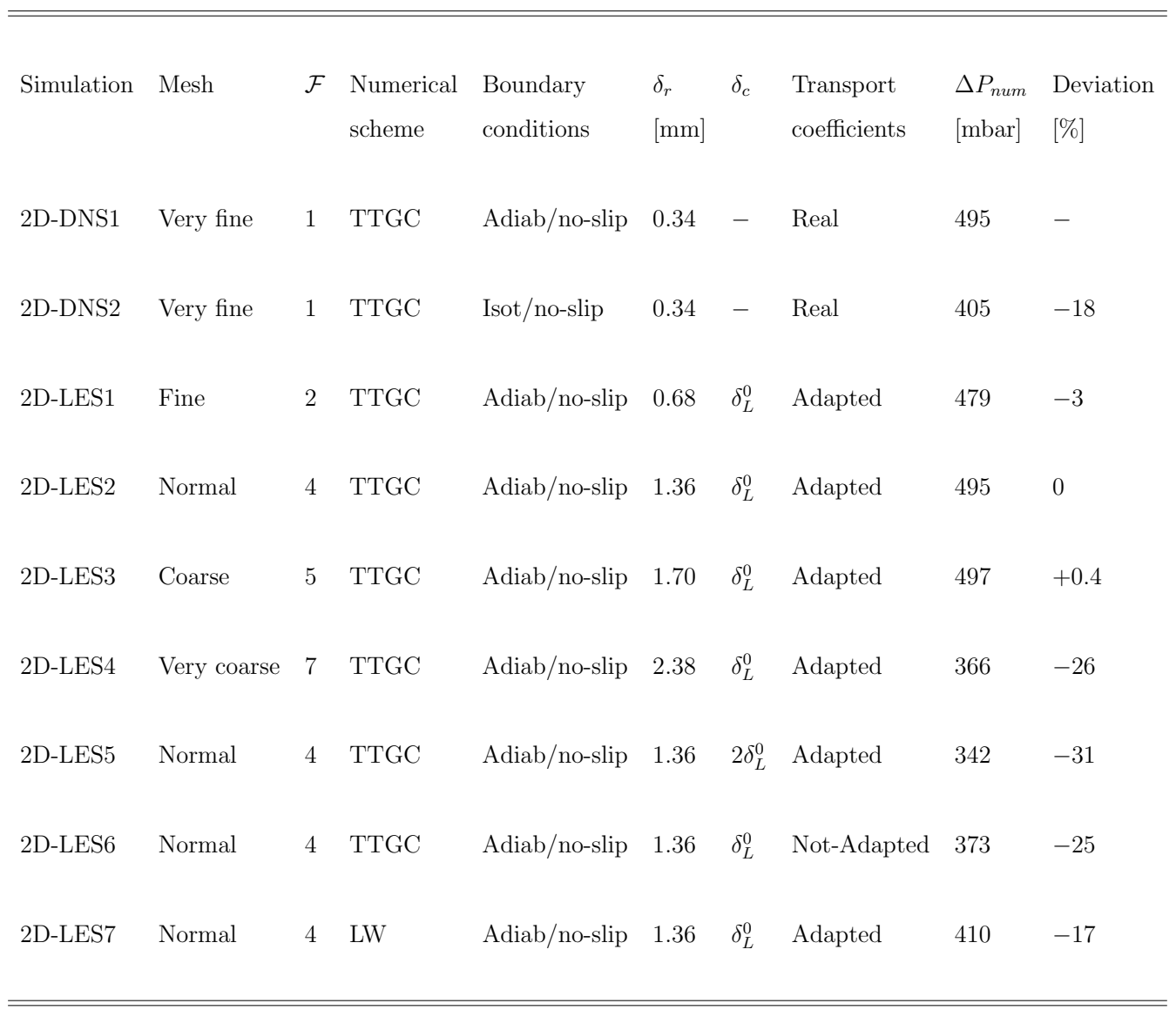

Table 5: Details of two-dimensional simulations and numerical parameters. $\mathcal{F}$ stands for the thickening factor, $\delta_{c}$ for the inner cut-off and $\Delta P_{\text {num }}$ for the maximum overpressure found numerically. TTGC: two-step Taylor-Galerkin scheme [63], LW: Lax-Wendroff scheme [64]. 


\begin{tabular}{lllll}
\hline \hline 2D meshes & $N_{\text {Cells }}[\mathrm{M}]$ & $N_{\text {Nodes }}[\mathrm{M}]$ & $\Delta_{x}[\mathrm{~mm}]$ & $\mathcal{F}$ \\
Very fine & 5.47 & 2.74 & 0.07 & 1 \\
Fine & 1.46 & 0.73 & 0.14 & 2 \\
& & & & \\
Normal & 0.35 & 0.18 & 0.28 & 4 \\
& & & & \\
Coarse & 0.24 & 0.12 & 0.34 & 5 \\
& & & & \\
Very coarse & 0.11 & 0.06 & 0.5 & 7 \\
\hline \hline
\end{tabular}

Table 6: Details of numerical meshes. $N_{\text {Cells }}$ stands for the number of cells and $N_{\text {nodes }}$ for the number of nodes. $\Delta_{x}$ denotes the grid spacing and $\mathcal{F}$ the thickening factor.

of wall conditions is investigated. For the atmosphere outlet, Navier-Stokes Characteristic Boundary conditions (NSCBC) [65] are prescribed. The initial flame kernel radius is set to $r_{i}=10 \mathrm{~mm}$. This technique has been employed in other studies $[25,32]$ and presents the advantage of initializing the combustion process without any complex transient phase. For example, the distribution of temperature at a radius, $r$, from the ignition point is defined as: 


$$
\widetilde{T}(r)=T_{a d}\left\{1-\left[0.5+0.5 \tanh \left(\frac{r-r_{i}}{\delta_{r}}\right)\right]\right\}
$$

The temperature within the ignition radius is set to the adiabatic flame temperature $T_{a d}=2275 \mathrm{~K}$. The initial numerical flame thickness $\delta_{r}=\mathcal{F} \delta_{L}^{0}$. The thickening factor is set to $\mathcal{F}=n \Delta_{x} / \delta_{L}^{0}, n$ being the number of cells used to resolve the flame front ( $n=5$ in the present calculations).

Then, numerical aspects of the 2D-LES are investigated. The influence of mesh size, inner cut-off, transport coefficients and numerical schemes are considered. For the 2D-LES, the dynamic Smagorinsky model [38] closes the sub-grid stress tensors. This choice was made because the WALE [66] model, used in our 3D LES, is not conceived for 2D simulations.

\subsection{Analysis of the 2D-DNS1}

The flame propagation and the vorticity field are shown in Fig. 4 for different moments of the simulation 2D-DNS1 ( $t=4,4.5,5,5.5,6,6.5 \mathrm{~ms})$. At early stages, the flame is laminar. The kernel moves gradually through the first range of obstacles and the flame, perfectly symmetrical and still laminar, starts to roll up. The expanding gases generate vortex cores behind the baffle plates that interact with the flame front subsequently. After $t=5$ ms, the flow symmetry is broken. This flame induced flow field increases turbulence, which in turn feeds the combustion, increasing the flame speed and the pressure inside the domain. Vortices interact strongly with the flame front and are essential to capture the correct flame behavior. Note that, even at the initial moments, when the flame is still at the first grid, turbulent structures are generated in the wake of the other obstructions (Fig. 4a). 
This limits the interest of mesh adaptation techniques, i.e. refine only in the flame region and degenerate the mesh elsewhere. Therefore, to perform an accurate LES, the mesh should be sufficiently fine and homogeneous, in order to capture these phenomena that are inter-correlated.

\subsection{Influence of the boundary conditions}

The reference case assumes no-slip and adiabatic walls, once the deflagration takes place in a very short period of time ( $7 \mathrm{~ms}$ for the $2 \mathrm{D}$ case). Another test was made with isothermal walls at temperature $300 \mathrm{~K}$. A reduction of $18 \%$ of the maximum overpressure is reported (Fig. 5 and Table 7 ) and is linked to the fact that burnt gases are cooled by the lower wall temperature, influencing the expanding rate and, consequently, the pressure inside the combustion chamber. This effect should be amplified when dealing with three-dimensional configurations, due to a more significant surface to volume ratio. Therefore, simulations assuming adiabatic walls should predict higher pressures than simulations considering isothermal walls. We conclude that to predict the maximum overpressure inside an explosion chamber, boundary conditions must be specified with care.

\subsection{Influence of the mesh resolution}

The influence of the mesh resolution and, consequently, the thickening factor $\mathcal{F}$ is now analyzed $\left(\mathcal{F}=n \Delta_{x} / \delta_{L}^{0}\right.$, with $\left.n=5\right)$. Figure 6 shows the overpressure evolution inside the combustion chamber for different values

of $\mathcal{F}$. For $\mathcal{F} \leq 5$, the reference overpressure obtained from DNS is well predicted by the LES, whereas for $\mathcal{F}=7$, the mesh is too coarse to capture properly the dynamics of the flow. As a matter of fact, two key points must 
be highlighted: (i) the mesh has to be reasonably fine so that two flame fronts can pass between the obstacles (see Fig. 7) and (ii) it needs to reproduce the essential vortex structures of the wakes. Considering the first point, if the flame front must be resolved within 5 grid points, a mesh size of $\Delta_{x}=0.5$ $\mathrm{mm}$, resulting in 10 points between the strips, is clearly not sufficient to carry out an accurate LES. The 2D-LES indicates that at least 15 points is needed between each grid. For the second point, Fig. 7 shows that the vorticity intensity is decreased, when using the coarsest mesh $(\mathcal{F}=7)$. As discussed in subsection 5.2, one cannot simulate correctly this type of flow if the vortices are mispredicted. The mesh corresponding to $\mathcal{F}=5$ is still able to capture the vortices behind the obstacles and is kept to run the 3D simulations.

Note that the pressure peak instant do not match and depends on the simulation. This point is linked to the fact that the response time of the flame is not the same for different thickening factors. There is also the response of the dynamic model, which by construction forces the unresolved scales to respond as the resolved ones. The unsteady response of the dynamic model will be studied in a near future and is not treated in this work. For these reasons, LES results are only based on the magnitude of the overpressure signal.

\subsection{Influence of the numerical scheme}

The third order TTGC scheme (2D-LES2) is compared with the second order Lax-Wendroff (2D-LES7) convective scheme in Fig. 8. The importance of a high order numerical scheme is clear when dealing with large eddy simulations, especially in this transient configuration, where the propaga- 
tion of the information is crucial and dissipative and dispersive errors may affect negatively the results. As shown in Fig. 8, using a less precise numerical scheme may lead to an under-estimation of $17 \%$ of the maximum overpressure. We conclude that high order schemes are essential to predict the maximum overpressure in this type of flow (at least for the present mesh).

\subsection{Influence of the inner cut-off length scale $\delta_{c}$}

The inner cut-off length scale $\delta_{c}$ plays an important role in the prediction of the maximum overpressure, as shown in Fig. 8. Setting $\delta_{c}=\delta_{L}^{0}$, the 2DLES2 give good results, if compared to the 2D-DNS1. On the other hand, changing $\delta_{c}=2 \delta_{L}^{0}$ (2D-LES5) highly impacts the results and the maximum pressure value is underestimated by about $30 \%$ (Table 5 ).

Note that $\delta_{c}$, which is the minimum curvature radius of the flame front, cannot be determined from a dynamic procedure, once it corresponds to scales lost in the filtering process $\left(\Delta>\delta_{c}\right)$. Volpiani et al. [46] showed the influence of this parameter by simulating a turbulent jet flame. Veynante and Moureau [50] carried out a priori analysis and showed that the inner cut-off length scale is close to the laminar flame thickness, in agreement with the present study. Gülder and Smallwood [53] suggested that the inner cut-off length scale may depend on the Karlovitz number. A more refined model for this physical parameter requires modeling of additional terms, such as the sub-grid scale turbulence intensity, and a simpler formulation is preferred here. 


\subsection{Influence of the transport modeling}

Figure 8 shows the overpressure evolution inside the combustion chamber for simulations 2D-DNS1 (reference), 2D-LES2 $(\mathcal{F}=4$ with modified transport coefficients) and 2D-LES6 $(\mathcal{F}=4$ without modified transport coefficients). In accordance with [59] and Fig. 3, results indicate that an adapted transport model reproducing the response of the thickened flame to stretch is essential to capture the correct overpressure. All 3D simulations have been carried out using the adapted transport model.

\section{Numerical set-up for the 3D LES}

The three-dimensional computational domain comprises the combustion chamber and a plenum attached to its open end. This plenum, which mimics the atmosphere, allows a better handling of acoustic waves, especially after the peak pressure and avoids imposing a boundary condition at the exit plane of the chamber. Simulations are performed with the two-step Taylor-Galerkin weighted residual central distribution scheme, third order in time and space (TTGC) [63] that complies with the necessity of low-dissipation for LES. The walls of the chamber and obstacles are modeled using isothermal law of the wall [67]. This type of boundary condition have also been employed in other studies by Masri and co-workers [17-19]. Nevertheless, a comparison with adiabatic no-slip walls classically used in this kind of configuration [14-16] is also performed. For the atmosphere outlet, Navier-Stokes Characteristic Boundary conditions (NSCBC) [65] are prescribed. The Wall Adapting Local Eddy (WALE) model [66] describes sub-grid stress tensors. Abdel-Raheem et al. [68] investigated the influence of initial conditions in the same combus- 
tion chamber (BBBS) for a premixed hydrogen/air mixture. The shape of the ignition kernel was varied from hemispherical to spherical and the ignition energy was varied by increasing the radius from 2 to $5 \mathrm{~mm}$. They concluded that the predicted overpressure is independent of the ignition radius and that the only change is in the timing of the occurrence of the pressure peak. Thus, results are not affected at least for $r_{i} \leq 5 \mathrm{~mm}$. In our 3D cases, calculations are initialized by a small sphere of burnt gases (radius $5 \mathrm{~mm}$ ) at the ignition point (Fig. 9).

The LES mesh contains about $60 \mathrm{M}$ cells/11M nodes and the typical cell size inside the chamber is $\Delta_{x} \approx 0.35 \mathrm{~mm}$, corresponding to a thickening factor $\mathcal{F} \approx 5$. The choice of the grid spacing takes into consideration the extensive study concerning the bi-dimensional test case.

The total wall clock simulation time is about $60 \mathrm{~h}$ on 4096 cores of an IBM BlueGene/Q machine for the 3D LES cases.

\section{A posteriori tests on the 3D LES of the small-scale experiment}

Numerical results are now compared to experimental data for three different geometries. They are discussed in terms of flow field visualization, over-pressure, flame surface and model parameter evolutions.

The overpressure signal is extracted from a probe located at the closed end of the chamber, as in experiments (indicated in Fig. 1). Note that LES results are not discussed in terms of timing criteria but only on the magnitude and trend of the overpressure signal. Firstly, because numerical parameters such as the initial flame kernel radius influence the timing of the peak location. Secondly, because of the variability of the experimental results. Different 
experimental realizations provided similar results for the maximum pressure and its rate of change but larger variations were observed for the time to reach the pressure peak. Therefore, in order to overcome experimental and numerical uncertainties, experimental signals have been shifted in time to match the LES peak. The same procedure is used experimentally.

Some definitions are now introduced. Resolved $\left(S_{r}\right)$ and total $\left(S_{t}\right)$ flame surfaces are estimated here as [46]:

$$
\begin{gathered}
S_{r}(t)=\int_{\mathcal{V}}|\nabla \widetilde{c}| d \mathcal{V} \\
S_{t}(t)=\int_{\mathcal{V}} \Xi_{\Delta}|\nabla \widetilde{c}| d \mathcal{V}
\end{gathered}
$$

The temporal evolution of these quantities provides information on the flame dynamics. The spatially averaged $\bar{\beta}$ parameter is defined as:

$$
\bar{\beta}(t)=\frac{\int_{\mathcal{V}_{f}} \beta(x, y, z, t) d \mathcal{V}}{\int_{\mathcal{V}_{f}} d \mathcal{V}}
$$

where $\mathcal{V}_{f}$ denotes the flame volume defined as $0.05 \leq \widetilde{c} \leq 0.95$.

\subsection{Configuration $O O B S$}

Figure 10 presents snapshots of the flame front corresponding to $\widetilde{c}=0.5$ colored by the model parameter for case OOBS. In the early stages of the flame development, when the flame is still laminar, the model parameter is close to zero as expected. When the flame starts to interact with the turbulence generated by the obstacles, the model exponent takes larger values depending on the local flame wrinkling. Differently from classical non-dynamic models which, most of the time, need some kind of tuning [16], the proposed 
local dynamic formulation adjusts the model parameter automatically on the fly as evidenced in Fig 10.

Figure 11 displays a sequence of images of the flame development in the middle plane $y=0$. At the early stages, the flame is laminar and propagates with a semi-spherical shape. The expanding gases generate vortex cores behind the baffle plates that interact with the flame front subsequently (as discussed in the previous section). Finger-like structures are generated when the flame crosses the first grid. Finally, this flame induced flow field increases turbulence and combustion intensity.

Figure 12 shows the temporal evolution of the overpressure together with the experimental envelope for case OOBS. The LES reproduces the overpressure peak and oscillations very well.

At early stages of the flame development, when the flame is not yet wrinkled by turbulence motions, resolved and total flame surfaces are equal and the model parameter is expected to be zero. As turbulence is generated, the sub-grid wrinkling factor increases to take into account the non-resolved flame surface. These observations are confirmed in Figs. 13 and 14 that display, respectively, the evolution of flame surfaces and mean model parameter as a function of the flame position defined as the maximum downstream $x$-location where the flame front is present. Passing the square obstacle, the flame accelerates and sub-grid wrinkling is detected automatically by the dynamic wrinkling factor model.

The mean parameter $\bar{\beta}$ remains close to zero until the moment the flame reaches the grid. The slight decrease of $\bar{\beta}$ at the beginning of the simulation is linked to the initial conditions and because the model predicts some wrinkling 
for small spherical laminar flame kernels [37]. Thereafter, it takes higher values and stabilizes around $\bar{\beta} \approx 0.3$. Note the strong flame acceleration. The flame takes $14.3 \mathrm{~ms}$ to move $0.10 \mathrm{~m}$ and achieve the central obstacle. On the other hand, after turbulent transition, it takes less than $2 \mathrm{~ms}$ to travel the same distance (from $x=0.10$ to $x=0.20 \mathrm{~m}$ ).

\subsection{Configuration $B B B S$}

The flame front corresponding to $\widetilde{c}=0.5$ colored by the model parameter for configuration BBBS is shown in Fig. 15. Compared to the other geometries, case BBBS combines three baffle plates and the square obstacle, leading to the largest turbulent intensity. The turbulent transition takes place when the flame reaches the second baffle plate. At this moment, the model parameter takes higher values. The flame is then strongly accelerated, resulting in a huge overpressure. According to Fig. 15, $\beta$ may locally take values of the order of, or slightly above unity, that would correspond to unphysical fractal dimensions. However, as pointed out in Section 2.2, Eq. (2), where $\beta$ varies both in space and time, is more general than a simple fractal model [31].

Figure 16 shows instantaneous snapshots of iso-contours of $\widetilde{c}=0.3$ and $\widetilde{c}=0.7$ for configuration BBBS. When the flame reaches the first baffle plate, it passes first through the two central passages and then through the lateral passages. At this point, the flame is practically laminar, as the turbulence generated in the wake of the first obstacle is very low (as already shown by the 2D-DNS). Then, the flame develops four finger-like structures, which are wrinkled by turbulence motions after crossing the second grid. The flame is strongly accelerated after passing the last baffle plate and the square obstacle.

The temporal evolution of the overpressure signal is plotted against ex- 
perimental measurements in Fig. 17. The LES is able to reproduce the overpressure magnitude with good accuracy. Similarly to the previous case, post-maximum overpressure oscillations are correctly captured as well. As expected, this configuration presents the strongest overpressure among the three studied cases. This is due to the high level of turbulence in the chamber, as already mentioned by Masri et al. [4].

Figure 18 shows the evolution of resolved and total flame surfaces as a function of the flame position. Resolved and total flame surfaces are similar until the flame reaches the second grid approximately. Then, turbulence is increased, and the dynamic wrinkling factor model captures the sub-grid flame surface. When the flame exits the chamber, the total surface is about twice the resolved flame surface.

In Fig. 19, the spatially averaged parameter, $\bar{\beta}$, is plotted as a function of the flame position. For case BBBS, the model parameter is progressively increased and it takes a constant value when flame/turbulence equilibrium is reached after the obstacles. At this point, the average parameter is close to $\bar{\beta} \approx 0.4$. This value is larger than the one found in the previous configuration $(\bar{\beta} \approx 0.3)$, highlighting the advantage of using a dynamic combustion model for this type of flow. Note that the flame takes about the same time as in configuration OOBS to reach $x=0.05 \mathrm{~m}$, then it accelerates brutally: 11.5 ms (against 14.3) for $x=0.10 \mathrm{~m}$ and 12.8 (against 16.1) for $x=0.20 \mathrm{~m}$.

\subsection{Configuration BOOS}

Figure 20 presents snapshots of the flame front corresponding to $\widetilde{c}=$ 0.5 colored by the model parameter for case BOOS. In this case, the flame relaminarizes after crossing the baffle plates placed near the ignition point. 
The model parameter remains close to zero even after the flame passes the central obstacle, indicating a laminar behavior.

Figure 21 shows LES images of the flame propagation for case BOOS. After passing the first baffle plate, the finger-like structures are generated as in case BBBS. However, the flame relaminarizes before the square obstacle, meaning that the turbulence intensity generated by the first baffle is not sufficient to accelerate the flame front. Even after passing the square obstacle, the flame front remains less wrinkled than cases OOBS and BBBS.

The time history of overpressure for the LES and experimental results is plotted in Fig. 22. The overpressure trend and its maximum value are in good agreement with experiments, although pressure is slightly over-predicted after crossing the baffle plate. As expected, configuration BOOS presents the lower overpressure among the three configurations. The present model predicts the maximum pressure value within the experimental envelope. Gubba et al. [19] obtained a value $41 \%$ greater than experiments (see Table 7), showing the challenge to correctly reproduce this configuration. For all other cases, they obtained good agreement with experimental data.

Figures 23 and 24 display the evolution of flame surfaces and mean model parameter as a function of the flame position, respectively. For case BOOS, the flame is well resolved and remains laminar even after crossing the first grid. Note that the model parameter increases after the first grid, but then it decreases close to zero, indicating the relaminarization of the flame (Fig. 24). Only at the end of the chamber, the flame accelerates and the present model detects some sub-grid wrinkling (Fig. 23). Note that, at the beginning, the flame goes barely faster than case OOBS, but at the end they leave the 
chamber exit practically at the same instant.

\subsection{Adiabatic vs Isothermal boundary conditions}

A secondary goal of this study is to point out that numerical results are very sensitive to boundary conditions and they have to be chosen with care. Adiabatic no-slip walls are easy to implement in a numerical code and are therefore largely employed to simulate deflagrating flames [14-16, 25]. Hence, computations assuming adiabatic no-slip walls are also performed and compared to isothermal walls using a law of the wall [67]. As a matter of fact, heat transfer may play an important role in this type of configuration, especially in the most turbulent geometry (BBBS) as confirmed by the two-dimensional DNS. Figure 25 shows that the correct overpressure is predicted only when using isothermal walls. When heat transfer is neglected, the thermal expansion of hot gases are much more intense, inducing a higher flame acceleration and overpressure.

Figure 26 shows the heat loss distribution as a function of the simulation time. Initially, from $t=7$ to $t=11.5 \mathrm{~ms}$ (see Fig. 16), heat transfer happens essentially through the obstruction walls. Heat losses through the chamber walls become important and of the same order of magnitude as the losses due to the baffle plates only when the flame is fully turbulent and passes the square obstacle. Around $t=13.1 \mathrm{~ms}$, the flame exits the combustion chamber. The heat released by the flame is plotted as a function of time in

Fig. 27. It can be seen that the percentage of heat losses due to the cold walls is around $5 \%$ of the total heat released. 


\begin{tabular}{llll}
\hline \hline & & & \\
Simulation & $\Delta P_{\text {exp }}$ & $\Delta P_{\text {num }}$ & $\Delta P_{\text {num }}[19]$ \\
LES-OOBS & $61-82$ & 80 & 71 \\
& & & \\
LES-BOOS & $21-41$ & 37 & 58 \\
& & & \\
LES-BBBS & $98-125$ & 138 & 125 \\
\hline \hline
\end{tabular}

Table 7: Summary of simulations and geometries studied. $\Delta P_{\exp }[\mathrm{mbar}]$ and $\Delta P_{\text {num }}[\mathrm{mbar}]$ stand for the maximum overpressure found experimentally and numerically, respectively. Values obtained by Gubba et al. [19] are also shown for comparison.

\subsection{Summary}

Table 7 summarizes the different cases studied. It also compares measurements [4] and numerical predictions given by the present study and the ones found in the literature [19]. The present dynamic model gives excellent results for all three simulated cases. In addition, with a formulation a priori simpler and cheaper than the dynamic scale-similarity FSD model [48], over-pressure predictions are as good as, or even better than the ones found by Gubba et al. [19].

Figure 28 compares total flame surfaces (Eq. 17) and spatially averaged $\bar{\beta}$ parameter (Eq. 18) as a function of the flame position for the three configurations. Evolutions of the model parameter strongly differ from one case 
to another while the total flame surface at the downstream location $x=0.25$ $\mathrm{m}$ is about twice for the most turbulent configuration BBBS compared to BOOS where flow relaminarizes. Interestingly, $\bar{\beta}$ evolutions are identical for these two configurations up to $x=0.05 \mathrm{~m}$, reached after about $10 \mathrm{~ms}$ (see Figs. 19 and 24): both flames evolves in the turbulent field generated by the first baffle. Then, the flame interacts with the second baffle in configuration BBBS, absent in configuration BOOS.

\section{Conclusion}

Simulations of turbulent premixed flames propagating past repeated obstacles are performed and the dynamic wrinkling factor model is validated using a posteriori analysis. Three distinct configurations studied experimentally by Masri et al. [4] are treated in this article. Configuration BBBS presents the larger number of obstacles, three grids followed by a small square obstruction, displaying the stronger overpressure. Case OOBS presents the second highest pressure and is characterized by a long laminar phase, once obstacles are placed far away of the ignition point. In case BOOS, after crossing the first baffle, the flame front is relaminarized before reaching the central obstacle leading to the lowest observed overpressure. Large eddy simulations reproduce all phases of the flame propagation extremely well and are in agreement with discussions presented in [4].

An extensive sensitivity analysis has also been carried out based on a twodimensional geometry. Important points of numerical simulations have been discussed: initial and boundary conditions, numerical schemes, grid refinements, transport coefficients and inner cut-offs. Using consistent information 
is vital to obtain accurate results.

The dynamic fractal-like model is able to capture both laminar and turbulent flame regimes. At early stages of the flame development, a laminar flame propagates in a flow essentially at rest and the model parameter is close to zero. Then, the flame front interacts with the obstacles, increasing turbulence and combustion intensity and the model parameter takes higher values at these stages. Hence, when using the dynamic formulation, the model parameter is computed on the fly and varies in time and location. Therefore, it presents a huge advantage if compared to classical non-dynamic models, as ad hoc tuning is no longer necessary. We conclude that LES is able to correctly estimate critical parameters of the explosion such as the overpressure and to improve understanding of this type of flows.

\section{Acknowledgments}

This work was performed using HPC resources of IDRIS under the allocation 2016-020164 made by GENCI (Grand Equipement National de Calcul Intensif). The authors would like to thank Prof. A. R. Masri for providing the experimental data.

\section{References}

[1] M. Fairweather, G. Hargrave, S. Ibrahim, D. Walker, Studies of premixed flame propagation in explosion tubes, Combust. Flame 116 (1999) $504-518$.

[2] J. Kent, A. Masri, S. Starner, S. Ibrahim, A new chamber to study premixed flame propagation past repeated obstacles, in: 5th Asia-Pacific 
conference on combustion, The University of Adelaide Australia, pp. 1720.

[3] R. Hall, A. Masri, P. Yaroshchyk, S. Ibrahim, Effects of position and frequency of obstacles on turbulent premixed propagating flames, Combust. Flame 156 (2009) 439-446.

[4] A. Masri, A. AlHarbi, S. Meares, S. Ibrahim, A comparative study of turbulent premixed flames propagating past repeated obstacles, Ind. Eng. Chem. Res. 51 (2012) 7690-7703.

[5] A. Masri, S. Ibrahim, N. Nehzat, A. Green, Experimental study of premixed flame propagation over various solid obstructions, Exp. Therm. Fluid Sci. 21 (2000) 109-116.

[6] E. S. Oran, V. N. Gamezo, Origins of the deflagration-to-detonation transition in gas-phase combustion, Combust. Flame 148 (2007) 4-47.

[7] G. Ciccarelli, S. Dorofeev, Flame acceleration and transition to detonation in ducts, Prog. Energy Combust. Sci. 34 (2008) 499-550.

[8] S. B. Dorofeev, Flame acceleration and explosion safety applications, Proc. Combust. Inst. 33 (2011) 2161-2175.

[9] J. Janicka, A. Sadiki, Large eddy simulation of turbulent combustion systems, Proc. Combust. Inst. 30 (2005) 537-547.

[10] H. Pitsch, Large-eddy simulation of turbulent combustion, Annu. Rev. Fluid Mech. 38 (2006) 453-482. 
[11] T. Poinsot, D. Veynante, Theoretical and numerical combustion, 3rd edition, 2011.

[12] L. Y. Gicquel, G. Staffelbach, T. Poinsot, Large eddy simulations of gaseous flames in gas turbine combustion chambers, Prog. Energy Combust. Sci. 38 (2012) 782-817.

[13] M. Kirkpatrick, S. Armfield, A. Masri, S. Ibrahim, Large eddy simulation of a propagating turbulent premixed flame, Flow Turbul. Combust. 70 (2003) 1-19.

[14] V. Di Sarli, A. Di Benedetto, G. Russo, S. Jarvis, E. J. Long, G. K. Hargrave, Large eddy simulation and PIV measurements of unsteady premixed flames accelerated by obstacles, Flow Turbul. Combust. 83 (2009) 227-250.

[15] V. Di Sarli, A. Di Benedetto, G. Russo, Using large eddy simulation for understanding vented gas explosions in the presence of obstacles, J. Hazard. Mater. 169 (2009) 435-442.

[16] V. Di Sarli, A. Di Benedetto, G. Russo, Sub-grid scale combustion models for large eddy simulation of unsteady premixed flame propagation around obstacles, J. Hazard. Mater. 180 (2010) 71-78.

[17] S. R. Gubba, S. S. Ibrahim, W. Malalasekera, A. R. Masri, Les modeling of premixed deflagrating flames in a small-scale vented explosion chamber with a series of solid obstructions, Combust. Sci. Technol. 180 (2008) 1936-1955. 
[18] S. R. Gubba, S. S. Ibrahim, W. Malalasekera, A. R. Masri, An assessment of large eddy simulations of premixed flames propagating past repeated obstacles, Combust. Theory Model. 13 (2009) 513-540.

[19] S. R. Gubba, S. S. Ibrahim, W. Malalasekera, A. R. Masri, Measurements and LES calculations of turbulent premixed flame propagation past repeated obstacles, Combust. Flame 158 (2011) 2465-2481.

[20] S. S. Ibrahim, S. R. Gubba, A. R. Masri, W. Malalasekera, Calculations of explosion deflagrating flames using a dynamic flame surface density model, J. Loss Prev. Process Ind. 22 (2009) 258-264.

[21] Di Sarli, V. and and Di Benedetto, A. and Russo, G., Large eddy simulation of transient premixed flame / vortex interactions in gas explosions, Chem. Eng. Sci. 71 (2012) 539-551.

[22] Di Sarli, V. and Di Benedetto, A., Sensitivity to the presence of the combustion submodel for large eddy simulation of transient premixed flame-vortex interactions, Ind. Eng. Chem. Res. 51 (2012) 7704-7712.

[23] Di Sarli, V. and Di Benedetto, A., Effects of non-equidiffusion on unsteady propagation of hydrogen-enriched methane/air premixed flames, Int. J. of Hydrog. Energy 38 (2013) 7510-7518.

[24] C. Johansen, G. Ciccarelli, Modeling the initial flame acceleration in an obstructed channel using large eddy simulation, J. Loss Prev. Process Ind. 26 (2013) 571-585.

[25] P. Quillatre, O. Vermorel, T. Poinsot, P. Ricoux, Large eddy simulation of vented deflagration, Ind. Eng. Chem. Res. 52 (2013) 11414-11423. 
[26] S. Patel, S. Jarvis, S. Ibrahim, G. Hargrave, An experimental and numerical investigation of premixed flame deflagration in a semiconfined explosion chamber, Proc. Combust. Inst. 29 (2002) 1849-1854.

[27] N. Peters, The turbulent burning velocity for large-scale and small-scale turbulence, J. Fluid Mech. 384 (1999) 107-132.

[28] H. Pitsch, L. D. De Lageneste, Large-eddy simulation of premixed turbulent combustion using a level-set approach, Proc. Combust. Inst. 29 (2002) 2001-2008.

[29] M. Boger, D. Veynante, H. Boughanem, A. Trouvé, Direct numerical simulation analysis of flame surface density concept for large eddy simulation of turbulent premixed combustion, in: Proc. Combust. Inst., volume 27, Elsevier, pp. 917-925.

[30] O. Colin, F. Ducros, D. Veynante, T. Poinsot, A thickened flame model for large eddy simulations of turbulent premixed combustion, Phys. Fluids 12 (2000) 1843-1863.

[31] F. Charlette, C. Meneveau, D. Veynante, A power-law flame wrinkling model for LES of premixed turbulent combustion part I: non-dynamic formulation and initial tests, Combust. Flame 131 (2002) 159-180.

[32] O. Vermorel, P. Quillatre, T. Poinsot, LES of explosions in venting chamber: a test case for premixed turbulent combustion models, Combust. Flame 183 (2017) 207-223.

[33] E. Hawkes, R. Cant, A flame surface density approach to large-eddy 
simulation of premixed turbulent combustion, Proc. Combust. Inst. 28 (2000) 51-58.

[34] S. Richard, O. Colin, O. Vermorel, A. Benkenida, C. Angelberger, D. Veynante, Towards large eddy simulation of combustion in spark ignition engines, Proc. Combust. Inst. 31 (2007) 3059-3066.

[35] O. Vermorel, S. Richard, O. Colin, C. Angelberger, A. Benkenida, D. Veynante, Towards the understanding of cyclic variability in a spark ignited engine using multi-cycle LES, Combust. Flame 156 (2009) 15251541.

[36] H. Weller, G. Tabor, A. Gosman, C. Fureby, Application of a flamewrinkling LES combustion model to a turbulent mixing layer, in: Proc. Combust. Inst., volume 27, Elsevier, pp. 899-907.

[37] S. Mouriaux, O. Colin, D. Veynante, Adaptation of a dynamic wrinkling model to an engine configuration, Proc. Combust. Inst. (2016).

[38] M. Germano, U. Piomelli, P. Moin, W. H. Cabot, A dynamic subgridscale eddy viscosity model, Phys. Fluids A 3 (1991) 1760-1765.

[39] F. Charlette, C. Meneveau, D. Veynante, A power-law flame wrinkling model for LES of premixed turbulent combustion part II: Dynamic formulation, Combust. Flame 131 (2002) 181-197.

[40] G. Wang, M. Boileau, D. Veynante, K. Truffin, Large eddy simulation of a growing turbulent premixed flame kernel using a dynamic flame surface density model, Combust. Flame 159 (2012) 2742-2754. 
[41] H. G. Im, T. S. Lund, J. H. Ferziger, Large eddy simulation of turbulent front propagation with dynamic subgrid models, Phys. Fluids 9 (1997) $3826-3833$.

[42] E. Knudsen, H. Pitsch, A dynamic model for the turbulent burning velocity for large eddy simulation of premixed combustion, Combust. Flame 154 (2008) 740-760.

[43] G. Wang, M. Boileau, D. Veynante, Implementation of a dynamic thickened flame model for large eddy simulations of turbulent premixed combustion, Combust. Flame 158 (2011) 2199-2213.

[44] T. Schmitt, A. Sadiki, B. Fiorina, D. Veynante, Impact of dynamic wrinkling model on the prediction accuracy using the F-TACLES combustion model in swirling premixed turbulent flames, Proc. Combust. Inst. 34 (2013) 1261-1268.

[45] T. Schmitt, M. Boileau, D. Veynante, Flame wrinkling factor dynamic modeling for large eddy simulations of turbulent premixed combustion, Flow Turbul. Combust. 94 (2015) 199-217.

[46] P. Volpiani, T. Schmitt, D. Veynante, A posteriori tests of a dynamic thickened flame model for large eddy simulations of turbulent premixed combustion, Combust. Flame 174 (2016) 166-178.

[47] P. S. Volpiani, T. Schmitt, D. Veynante, Large eddy simulation of a turbulent swirling premixed flame coupling the TFLES model with a dynamic wrinkling formulation, Combust. Flame 180 (2017) 124-135. 
[48] R. Knikker, D. Veynante, C. Meneveau, A dynamic flame surface density model for large eddy simulation of turbulent premixed combustion, Phys. Fluids 16 (2004) L91-L94.

[49] T. Butler, P. O'Rourke, A numerical method for two dimensional unsteady reacting flows, in: Proc. Combust. Inst., volume 16, Elsevier, pp. $1503-1515$.

[50] D. Veynante, V. Moureau, Analysis of dynamic models for large eddy simulations of turbulent premixed combustion, Combust. Flame 162 (2015) $4622-4642$.

[51] F. Gouldin, An application of fractals to modeling premixed turbulent flames, Combust. Flame 68 (1987) 249-266.

[52] F. Gouldin, K. Bray, J.-Y. Chen, Chemical closure model for fractal flamelets, Combust. Flame 77 (1989) 241-259.

[53] Ö. L. Gülder, G. J. Smallwood, Inner cutoff scale of flame surface wrinkling in turbulent premixed flames, Combust. Flame 103 (1995) $107-114$.

[54] V. Moureau, P. Domingo, L. Vervisch, From large-eddy simulation to direct numerical simulation of a lean premixed swirl flame: Filtered laminar flame-pdf modeling, Combust. Flame 158 (2011) 1340-1357.

[55] G. P. Smith, D. M. Golden, M. Frenklach, N. W. Moriarty, B. Eiteneer, M. Goldenberg, C. T. Bowman, R. K. Hanson, S. Song, W. C. Gardiner Jr, et al., Gri-mech 3.0, 1999. 
[56] W. B. Bush, F. E. Fendell, Asymptotic analysis of laminar flame propagation for general lewis numbers, Combust. Sci. Technol. 1 (1970) 421-428.

[57] P. Clavin, Dynamic behavior of premixed flame fronts in laminar and turbulent flows, Prog. Energy Combust. Sci. 11 (1985) 1-59.

[58] P. Clavin, G. Joulin, Premixed flames in large scale and high intensity turbulent flow, J. Physique Lett. 44 (1983) 1-12.

[59] P. Quillatre, O. Vermorel, T. Poinsot, Large eddy simulation of turbolent premixed flames propagation in a small scale venting chamber: influence of chemistry and transport modelling, in: Seventh Mediterranean Combustion Symposium, Sardinia.

[60] P. Quillatre, Simulation aux grandes échelles d'explosions en domaine semi-confiné, Ph.D. thesis, École Doctorale Mécanique, Énergétique, Génie civil et Procédés (Toulouse); 154236012, 2014.

[61] V. Giovangigli, Multicomponent flow modeling. modeling and simulation in science, engineering and technology, 1999.

[62] T. Schonfeld, M. Rudgyard, Steady and unsteady flow simulations using the hybrid flow solver avbp, AIAA J. 37 (1999) 1378-1385.

[63] O. Colin, M. Rudgyard, Development of high-order Taylor-Galerkin schemes for LES, J. Comput. Phys. 162 (2000) 338-371.

[64] P. D. Lax, B. Wendroff, Difference schemes for hyperbolic equations 
with high order of accuracy, Commun. Pure Appl. Math. 17 (1964) 381-398.

[65] T. J. Poinsot, S. Lele, Boundary conditions for direct simulations of compressible viscous flows, J. Comput. Phys. 101 (1992) 104-129.

[66] F. Nicoud, F. Ducros, Subgrid-scale stress modelling based on the square of the velocity gradient tensor, Flow Turbul. Combust. 62 (1999) 183200.

[67] B. Kader, Temperature and concentration profiles in fully turbulent boundary layers, Int. J. Heat Mass Tran. 24 (1981) 1541-1544.

[68] M. A. Abdel-Raheem, S. S. Ibrahim, W. Malalasekera, A. R. Masri, Large eddy simulation of hydrogen-air premixed flames in a small scale combustion chamber, Int. J. Hydrog. Energy 40 (2015) 3098-3109. 


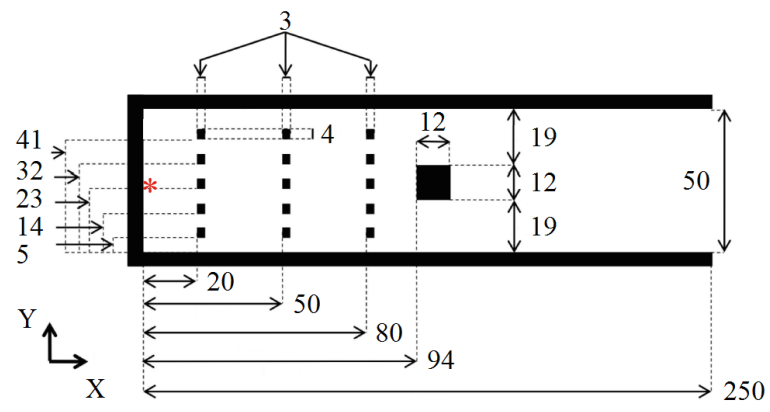

Figure 1: Top view of the studied combustion chamber [4]. Dimensions are in millimeters. The pressure transducer is located in the center of the closed end and is represented in the image by the symbol $*$.

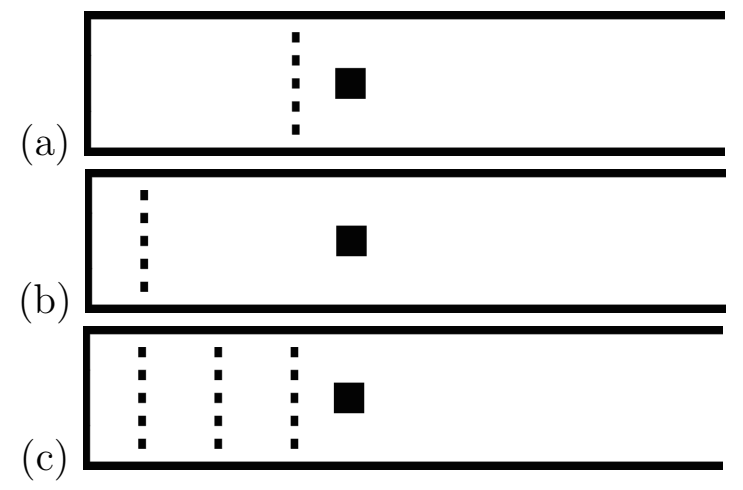

Figure 2: Studied configurations (a) OOBS, (b) BOOS and (c) BBBS. 


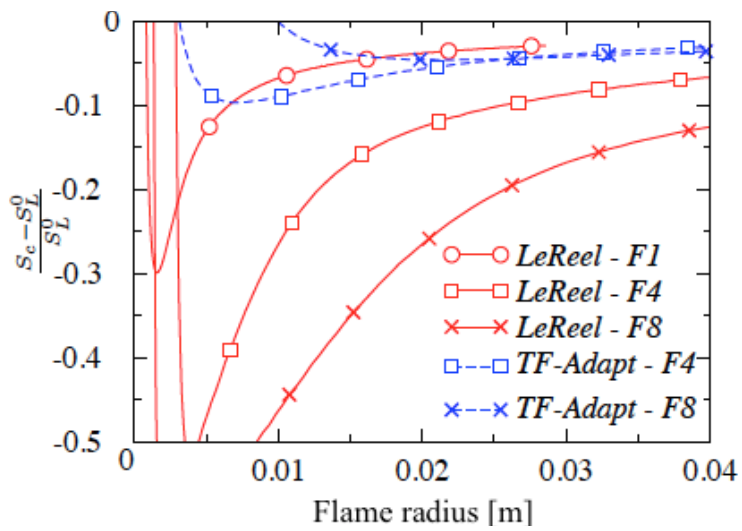

Figure 3: Normalized consumption speed as a function of the flame radius for different thickening factor with and without correction. Real transport coefficients $\mathcal{F}=1$ (- $\bigcirc)$, Transport coefficients without correction for $\mathcal{F}=4(-\times-)$ and $\mathcal{F}=8(-\square-)$, and with correction for $\mathcal{F}=4(-\square-)$ and $\mathcal{F}=8(-\bigcirc-)$. From Quillatre [60]. 
(a)

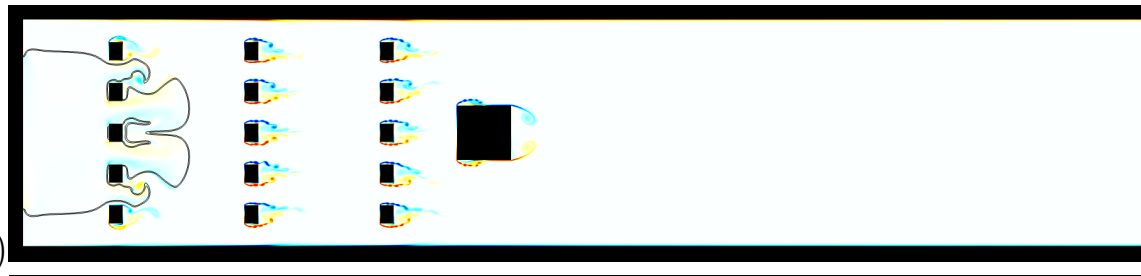

(b)
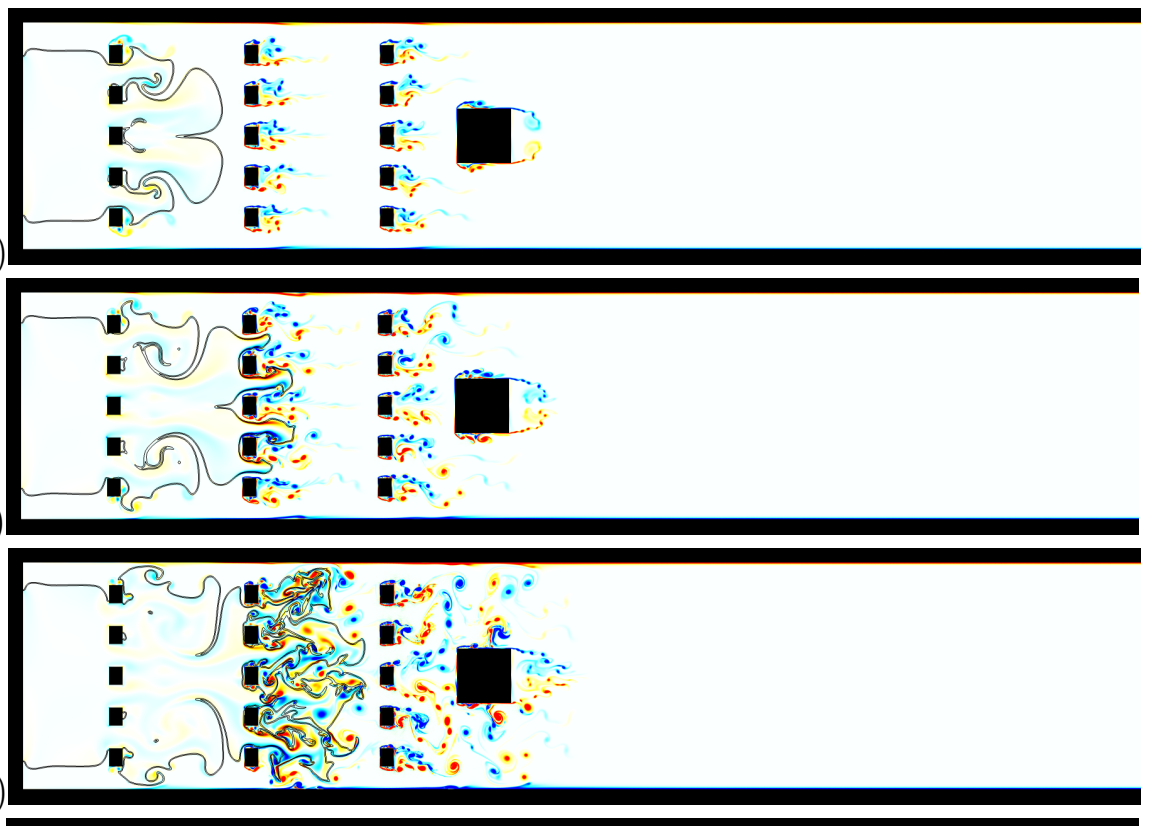

(d)

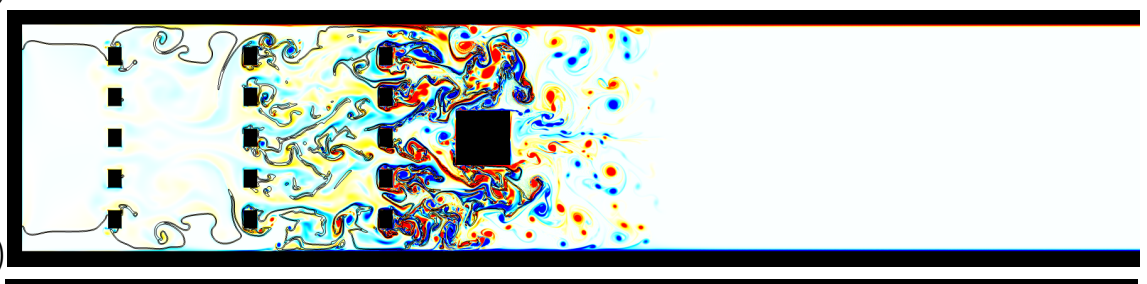

(e)

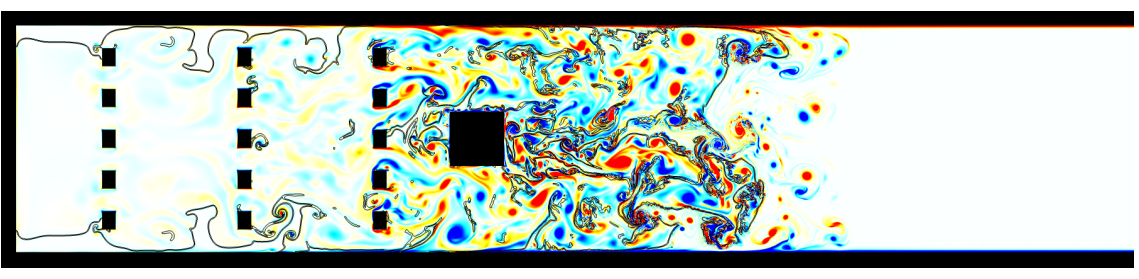

Figure 4: Simulation 2D-DNS1. Snapshots of the vorticity field with isocontours of the progress variable $(c=0.2$ and $c=0.8)$ that indicates the flame front for: (a) $t=4 \mathrm{~ms}$, (b) $t=4.5 \mathrm{~ms}$, (c) $t=5 \mathrm{~ms}$, (d) $t=5.5 \mathrm{ms,}$ (e) $t=6 \mathrm{~ms}$ and , (f) $t=6.5 \mathrm{~ms}$. The red color corresponds to $\Omega_{z}=5 \times 10^{4}$ $\mathrm{s}^{-1}$, while blue corresponds to $\Omega_{z}=-5 \times 10^{4} \mathrm{~s}^{-1}$. 


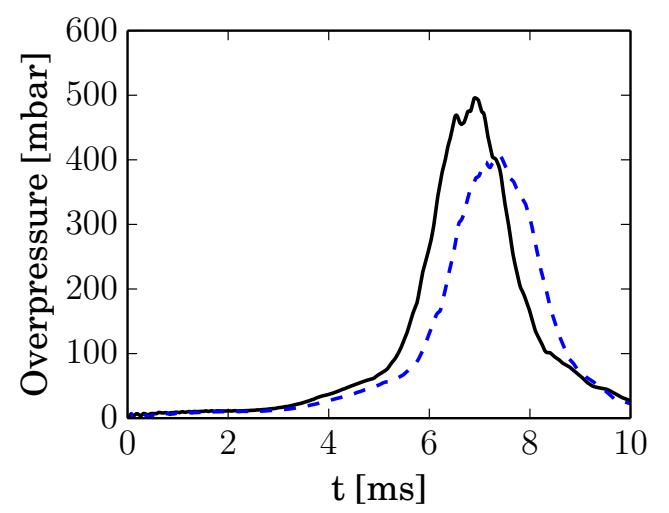

Figure 5: Comparison of overpressure signals for different 2D-DNS: () 2D-DNS1 (adiabatic/no-slip walls); (-- - ) 2D-DNS2 (isothermal/no-slip walls).

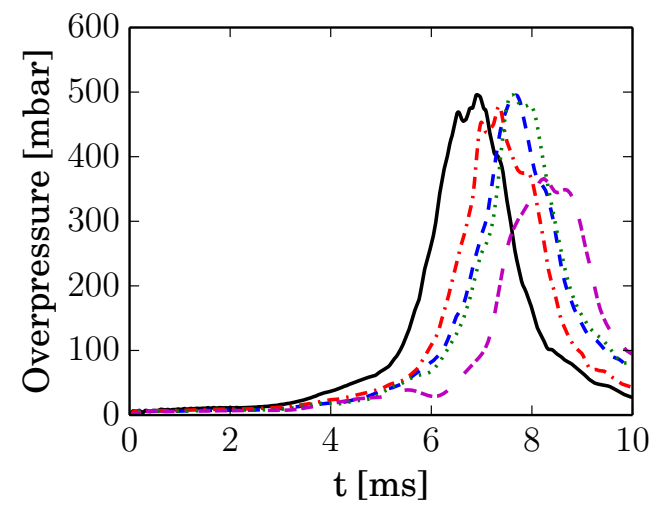

Figure 6: Comparison of overpressure signals for several 2D-LES using different mesh resolutions: $(-)$ 2D-DNS1 $(\mathcal{F}=1) ;(-\cdot-)$ 2D-LES1 $(\mathcal{F}=2),(-$ - - ) 2D-LES2 $(\mathcal{F}=4),(\cdots)$ 2D-LES3 $(\mathcal{F}=5),(---) 2 \operatorname{D-LES} 4(\mathcal{F}=7)$. 


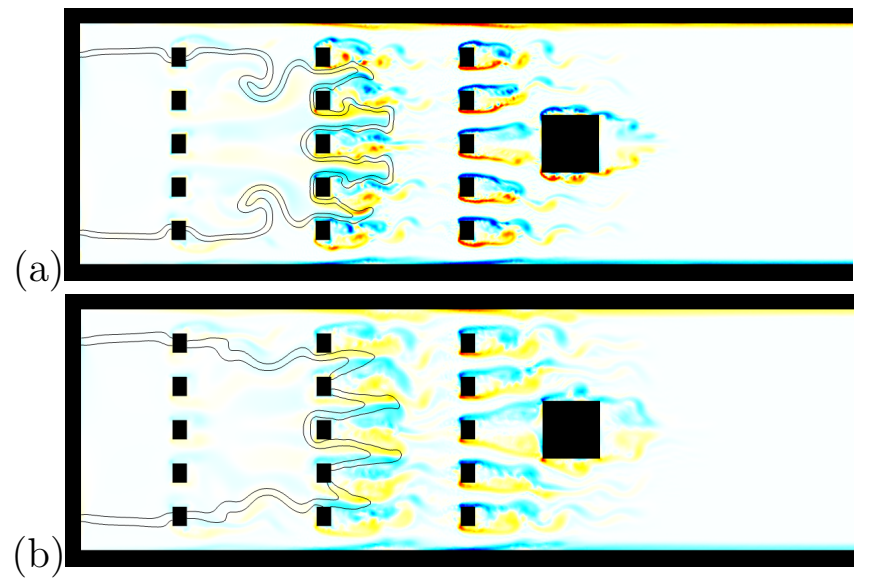

Figure 7: Snapshots of the vorticity field with iso-contours of the progress variable $(c=0.2$ and $c=0.8)$ that indicates the flame front for simulations: (a) 2D-LES3 $(\mathcal{F}=5, t=6 \mathrm{~ms})$, (b) 2D-LES4 $(\mathcal{F}=7, t=6.5 \mathrm{~ms})$. The red color corresponds to $\Omega_{z}=5 \times 10^{4} \mathrm{~s}^{-1}$, while blue corresponds to $\Omega_{z}=-5 \times 10^{4}$ $\mathrm{s}^{-1}$. 


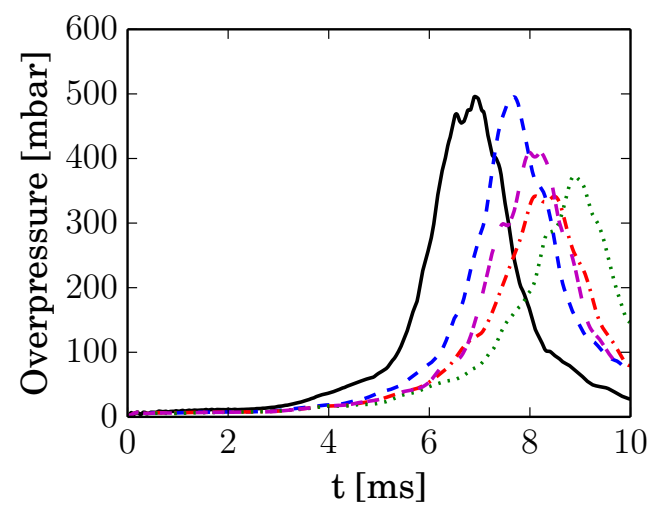

Figure 8: Comparison of overpressure signals for different simulations:

) 2D-DNS1; (-- - ) 2D-LES2 (TTGC, $\delta_{c}=\delta_{L}^{0}$, adapted transport coefficients), (-・- ) 2D-LES5 $\left(\delta_{c}=2 \delta_{L}^{0}\right) ;(\cdots)$ 2D-LES6 (non-adapted transport coefficients), (- - - ) 2D-LES7 (LW).

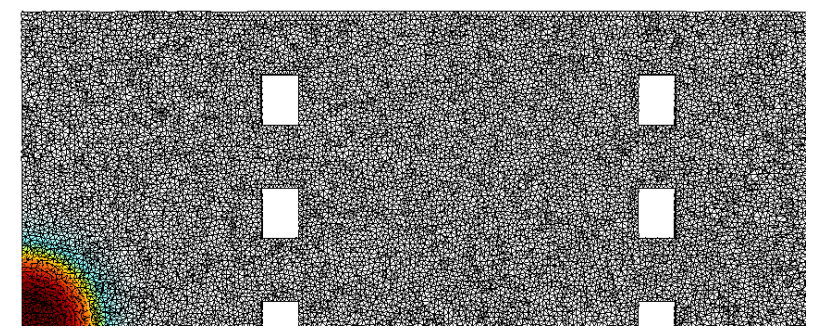

Figure 9: Zoom on the mesh and detail of the initial condition. The semi sphere in red indicates the region where temperature is higher than $1000 \mathrm{~K}$. For sake of clarity, only half of the $y=0$ plane is shown. 

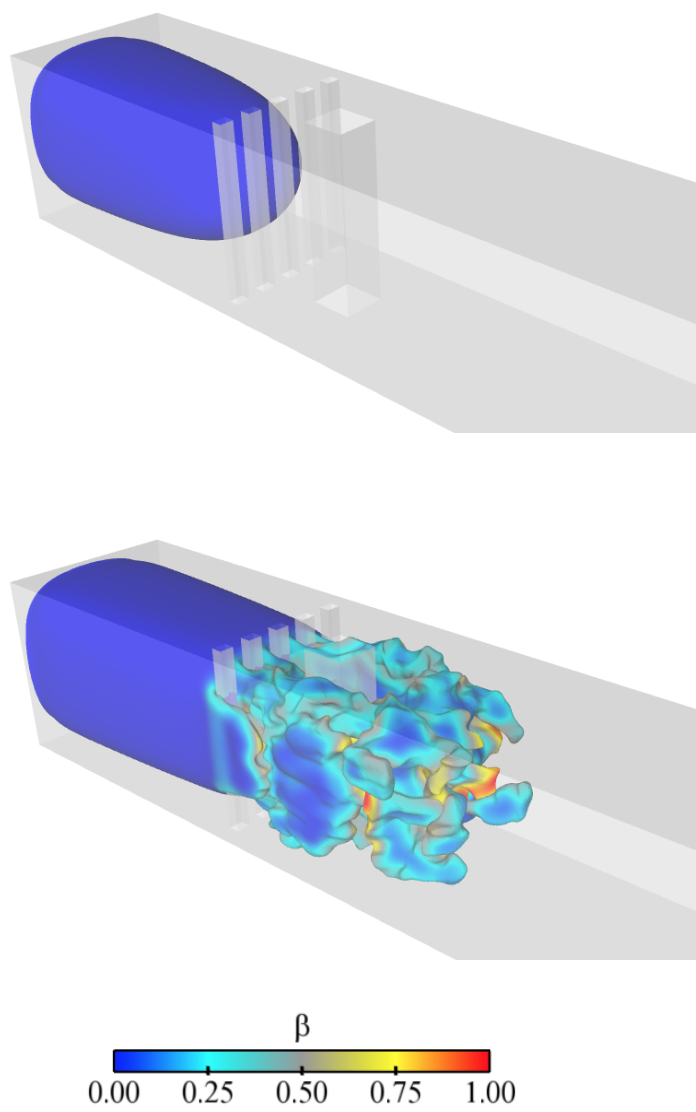

Figure 10: Instantaneous snapshots of the flame front corresponding to $\widetilde{c}=$ 0.5, obtained from simulation LES-OOBS, colored by the model parameter $\beta$. Instants correspond to $t=13.5 \mathrm{~ms}$ (top) and $t=15.5 \mathrm{~ms}$ (bottom). 


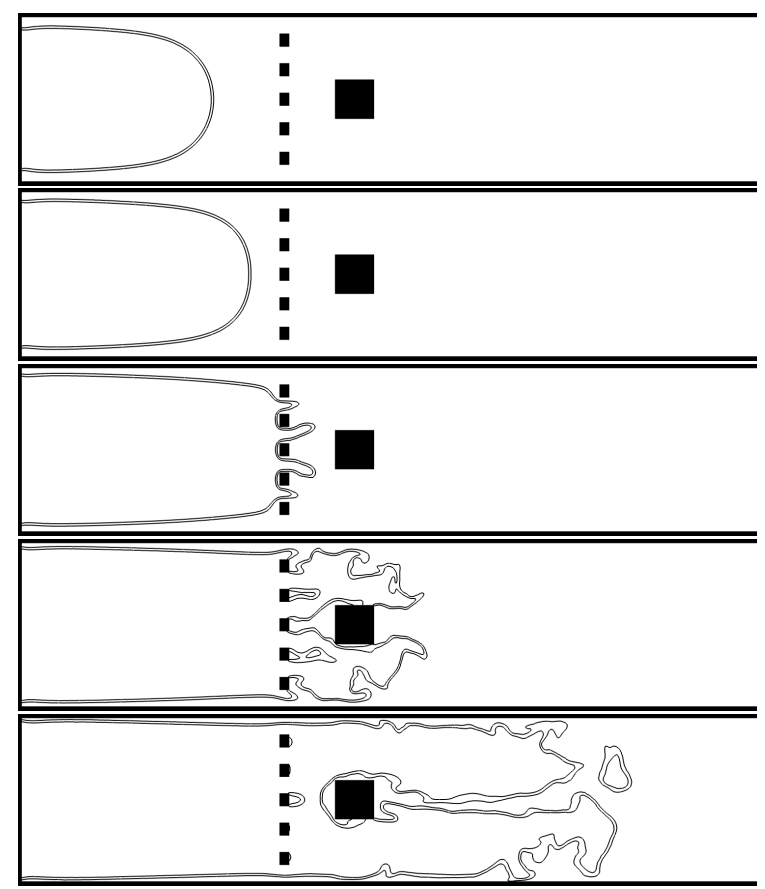

Figure 11: Instantaneous snapshots of the flame front represented by isocontours $\widetilde{c}=0.2$ and $\widetilde{c}=0.8$ for configuration OOBS. Instants correspond to $t=12,13,14,15$ and $16 \mathrm{~ms}$ (from top to bottom). 


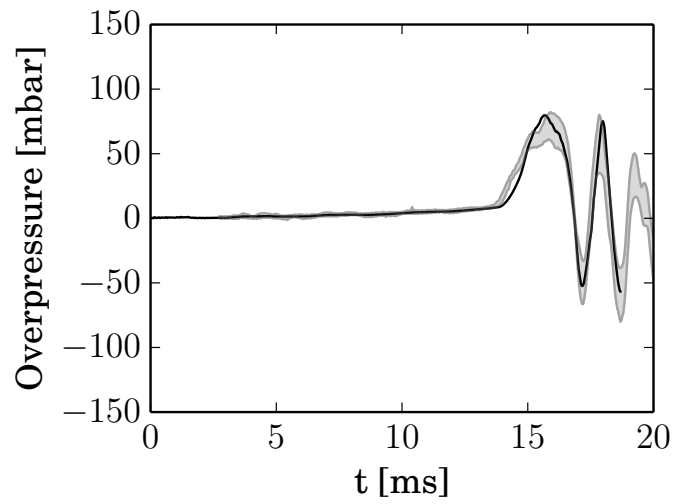

Figure 12: Comparison of overpressure signals between LES (solid line) and experiments [4] (grey envelope) for configuration OOBS. Note that the experimental envelope has been shifted in time to match the LES peak.

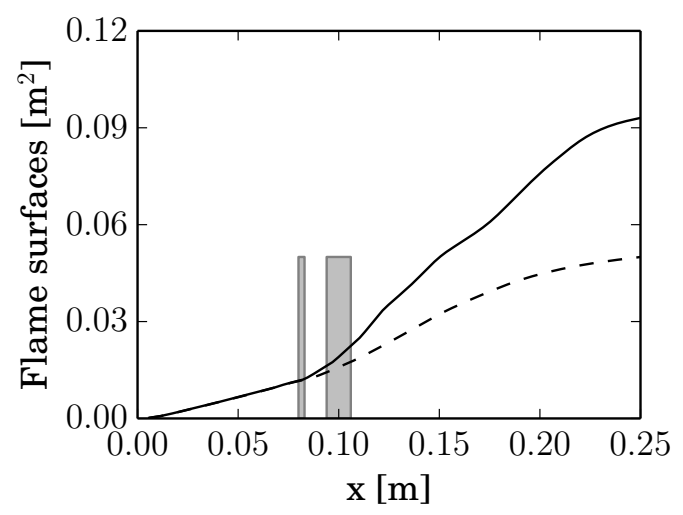

Figure 13: Evolution of resolved (Eq. 16, dashed line) and total (Eq. 17, solid line) flame surfaces as a function of the flame position for configuration OOBS. The grey rectangles display locations and thicknesses of the obstacles, here the third baffle and the square shaped central obstacle. 


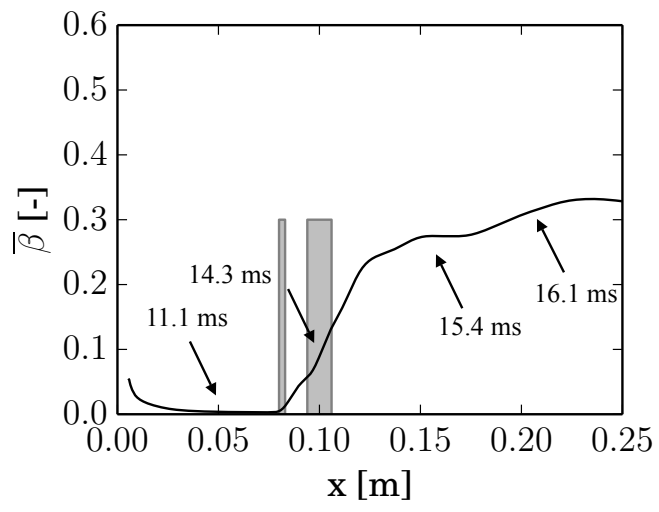

Figure 14: Evolution of the spatially averaged $\bar{\beta}$ parameter (Eq. 18) as a function of the flame position for configuration OOBS. The instants of the simulation are also indicated in the figure. The grey rectangles display locations and thicknesses of the third baffle and the square shaped central obstacle. 

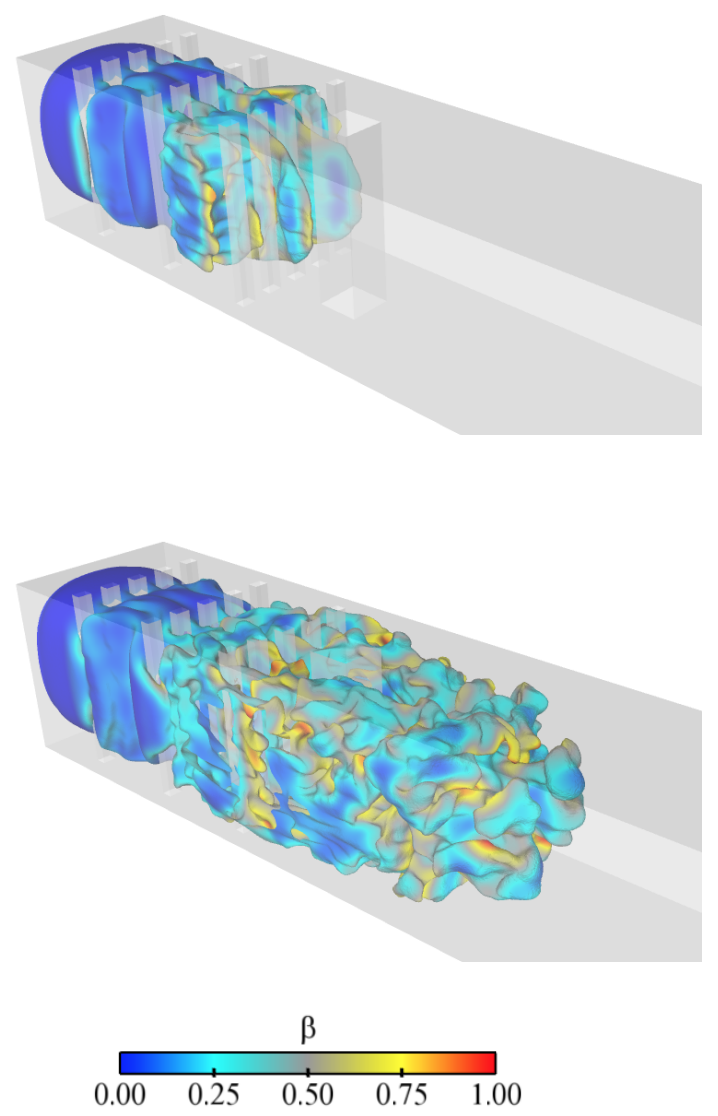

Figure 15: Instantaneous snapshots of the flame front corresponding to $\widetilde{c}=$ 0.5, obtained from simulation LES-BBBS, colored by the model parameter $\beta$. Instants correspond to $t=11.5 \mathrm{~ms}$ (top) and $t=12.5 \mathrm{~ms}$ (bottom). 


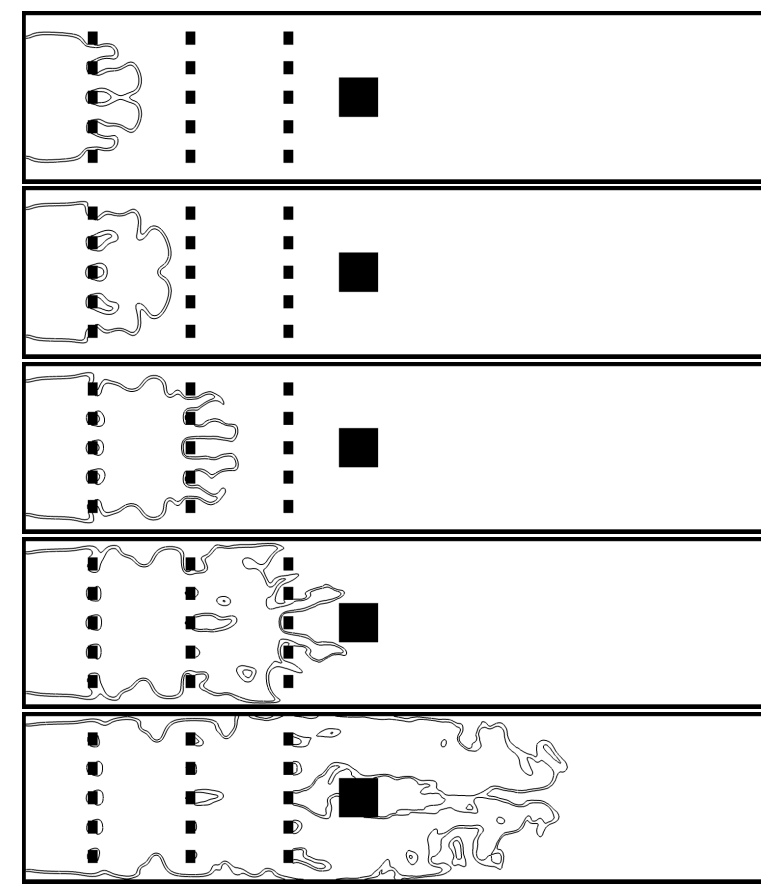

Figure 16: Instantaneous snapshots of the flame front represented by isocontours $\widetilde{c}=0.2$ and $\widetilde{c}=0.8$ for configuration BBBS. Instants correspond to $t=8.5,9.5,10.5,11.5$ and $12.5 \mathrm{~ms}$ (from top to bottom). 


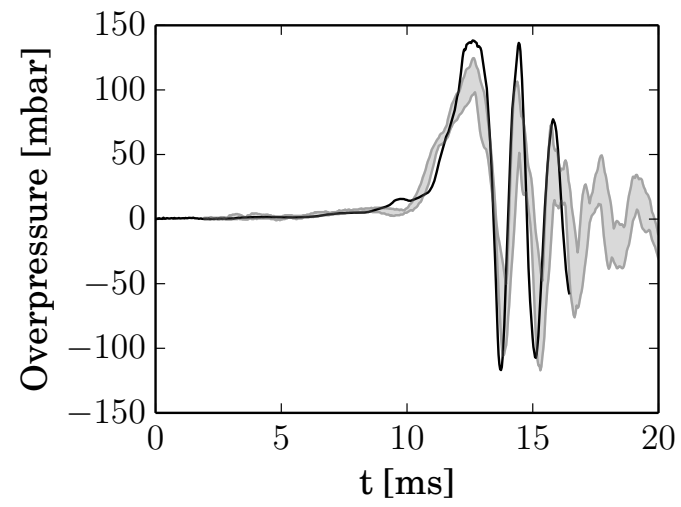

Figure 17: Comparison of overpressure signals between LES (solid line) and experiments [4] (grey envelope) for configuration BBBS.

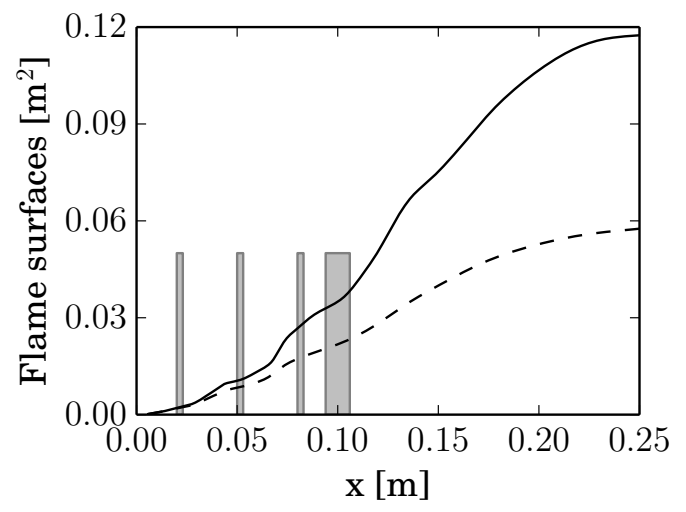

Figure 18: Evolution of resolved (Eq. 16, dashed line) and total (Eq. 17, solid line) flame surfaces as a function of the flame position for configuration BBBS. The grey rectangles correspond to the locations and thicknesses of the three baffles and the square shaped central obstacle. 


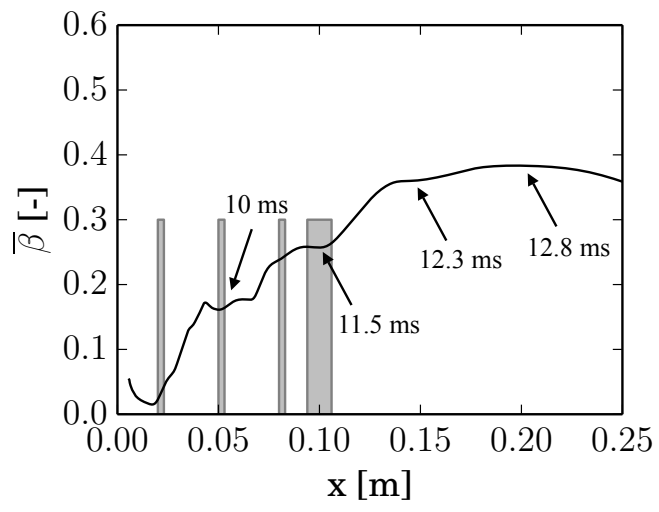

Figure 19: Evolution of the spatially averaged $\bar{\beta}$ parameter (Eq. 18) as a function of the flame position for configuration BBBS. The instants of the simulation are also indicated in the figure. The grey rectangles correspond to the locations and thicknesses of the three baffles and the square shaped central obstacle. 

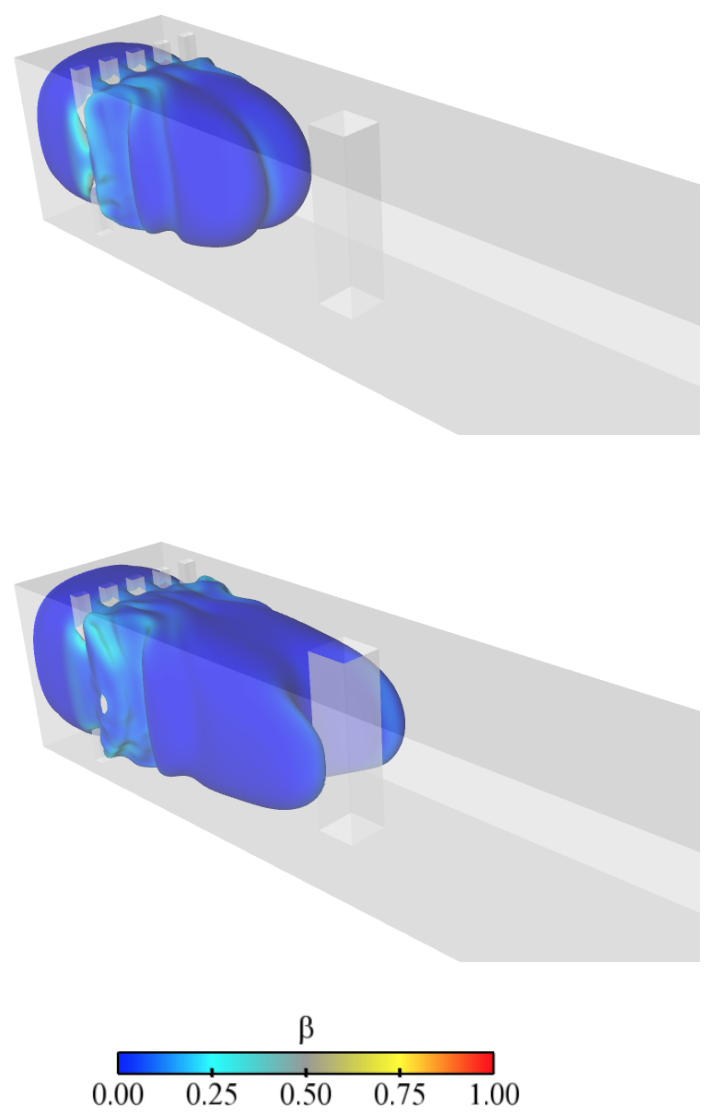

Figure 20: Instantaneous snapshots of the flame front corresponding to $\widetilde{c}=$ 0.5, obtained from simulation LES-BOOS, colored by the model parameter $\beta$. Instants correspond to $t=12 \mathrm{~ms}$ (top) and $t=13.5 \mathrm{~ms}$ (bottom). 


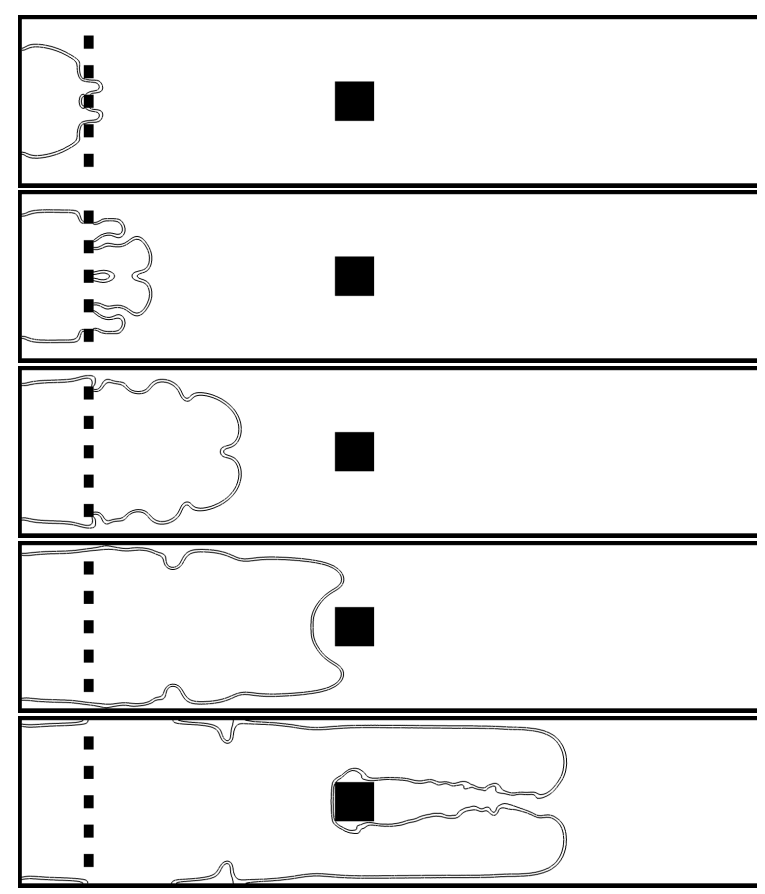

Figure 21: Instantaneous snapshots of the flame front represented by isocontours $\widetilde{c}=0.2$ and $\widetilde{c}=0.8$ for configuration BOOS. Instants correspond to $t=7,9,11,13$ and $15 \mathrm{~ms}$ (from top to bottom). 


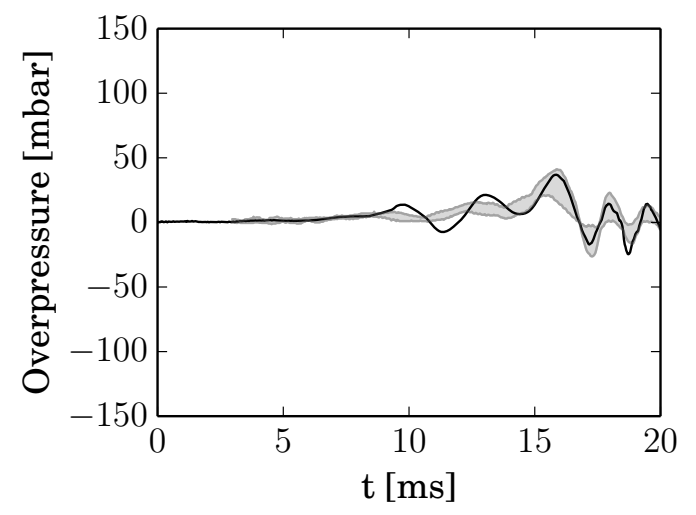

Figure 22: Comparison of overpressure signals between LES (solid line) and experiments [4] (grey envelope) for configuration BOOS.

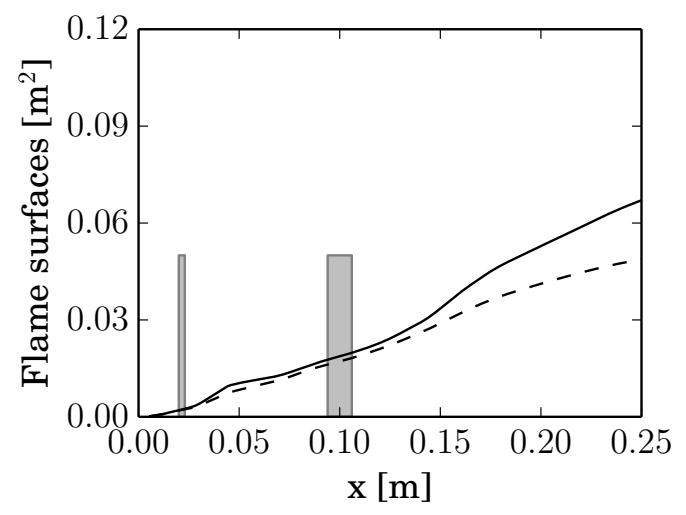

Figure 23: Evolution of resolved (Eq. 16, dashed line) and total (Eq. 17, solid line) flame surfaces as a function of the flame position for configuration BOOS. The grey rectangles give locations and thicknesses of the first baffle and the square shaped central obstacle. 


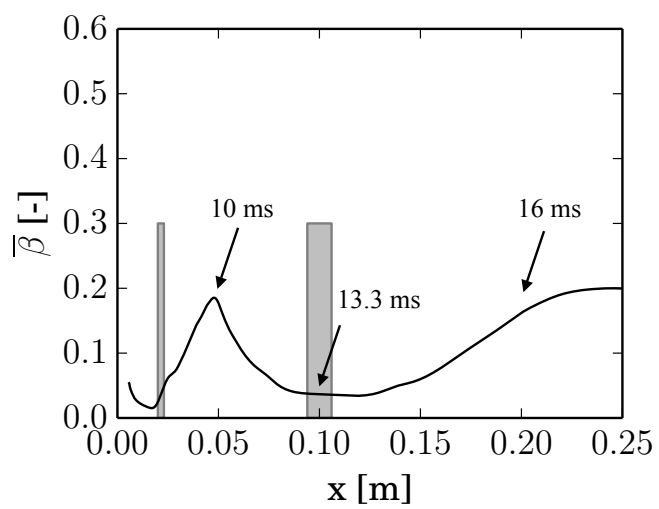

Figure 24: Evolution of the spatially averaged $\bar{\beta}$ parameter (Eq. 18) as a function of the flame position for configuration BOOS. The instants of the simulation are also indicated in the figure. The grey rectangles correspond to the locations and thicknesses of the first baffle and the square shaped central obstacle.

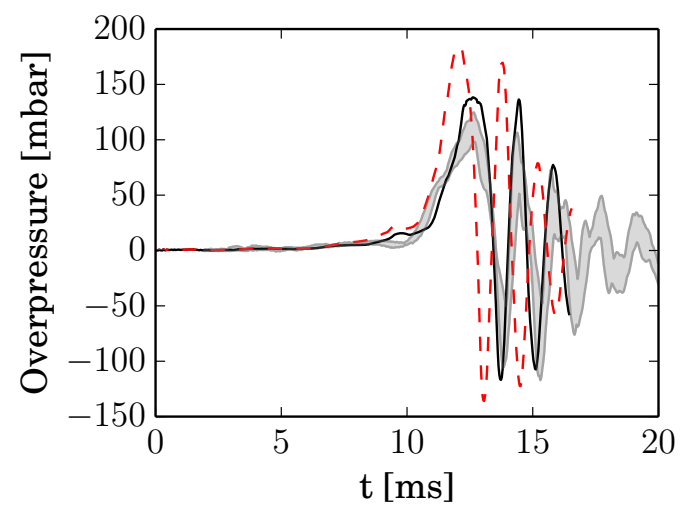

Figure 25: Comparison of overpressure signals between LES using isothermal law of the wall (solid line), LES using no-slip adiabatic walls (dashed line) and experiments (grey envelope) for configuration BBBS. 


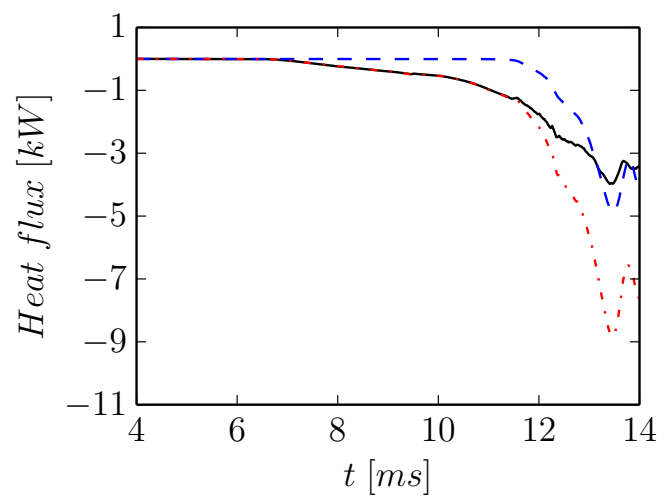

Figure 26: Heat fluxes as a function of simulation time: ( - ) heat flux through obstruction walls; (- - - ) heat flux through chamber walls and (- . - ) sum of heat fluxes.

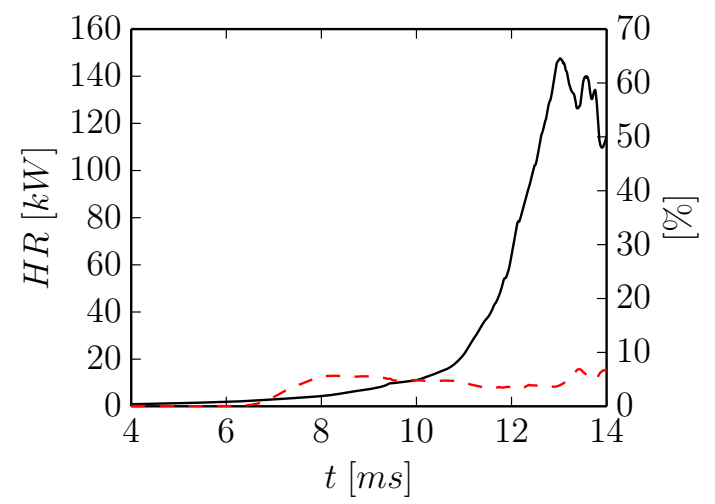

Figure 27: Heat released by the flame as a function of time ( - ) and percentage lost by the cold walls (-- - ). 

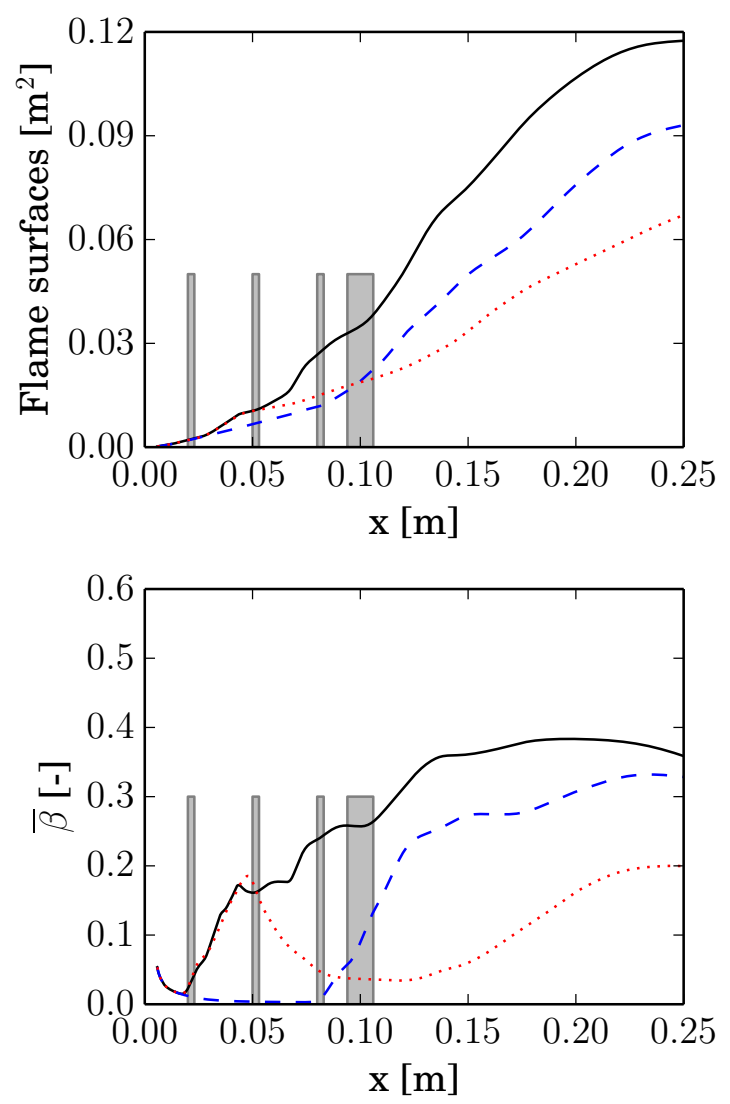

Figure 28: Evolution of the total flame surfaces (top) and the spatially averaged $\bar{\beta}$ parameter (bottom) s a function of the flame position for configurations OOBS (dashed line), BOOS (dotted line) and BBBS (solid line). The grey rectangles give locations and thicknesses of the three baffles (all present only in the BBBS case) and the square shaped obstacles. 